\title{
THE EXPLICIT GREEN'S APPROACH WITH STABILITY ENHANCEMENT FOR SOLVING THE BIOHEAT TRANSFER EQUATION
}

\author{
F.S. Loureiro ${ }^{\mathrm{a}, \mathrm{d}^{*}}$, W.J. Mansur ${ }^{\mathrm{b}}$, L.C. Wrobel ${ }^{\mathrm{c}}$ and J. E. A. Silva ${ }^{\mathrm{d}}$ \\ ${ }^{a}$ Department of Computer Science, Federal University of Juiz de Fora,36036-330, Juiz \\ de Fora, MG, Brazil \\ ${ }^{b}$ Department of Civil Engineering, COPPE/Federal University of Rio de Janeiro, \\ 21945-970, Rio de Janeiro, RJ, Brazil \\ ${ }^{c}$ School of Engineering and Design, Brunel University, UB8 3PH , Uxbridge, London, \\ $U K$ \\ ${ }^{d}$ Postgraduate Program in Computational Modeling, Federal University of Juiz de \\ Fora, 36036-900, Juiz de Fora, MG, Brazil \\ *Corresponding author. Tel.: +55 32 3229-381; \\ E-mail address: felipe.loureiro@ufjf.edu.br (F. S. Loureiro).
}




\begin{abstract}
The aim of this paper is to propose a strategy for performing a stability enhancement into the Explicit Green's Approach (ExGA) method applied to the bioheat transfer equation. The ExGA method is a time-stepping technique that uses numerical Green's functions in the time domain; these functions are here computed by the FEM. Basically, a new two nonequal time substeps procedure is proposed to compute Green's functions at the first time step. This is accomplished by adopting the standard explicit Euler scheme and an optimized procedure to yield the best stability constraint, allowing a reduction into the number of time steps without loss of accuracy. In addition, the concept of local numerical Green's functions is introduced and explored aiming at reducing the computational effort of nodal Green's functions calculation. Two examples are presented in order to show the potentialities of the proposed methodology, one to illustrate the accuracy and another applied to skin burn simulations.
\end{abstract}

Keywords: numerical Green’s functions; FEM; time substeps; Pennes bioheat equation; time integration; skin burns 


\begin{tabular}{|c|c|c|c|}
\hline \multicolumn{4}{|c|}{ Nomenclature } \\
\hline$c$ & specific heat $\left(\mathrm{Jkg}^{-10} \mathrm{C}^{-1}\right)$ & Greek symbols & \\
\hline $\mathbf{F}(t)$ & external heat load vector & $\Omega^{h}$ & discrete domain \\
\hline $\mathbf{G}(t)$ & Green's matrix & $\Omega_{G_{j}}^{h}$ & $\begin{array}{l}\text { discrete local } \\
\text { domain around } \mathbf{y}_{j}\end{array}$ \\
\hline$G(\mathbf{x}, \mathbf{y}, t-\tau)$ & Green's function & $\alpha_{i} \Delta t$ & substeps \\
\hline $\mathbf{I}$ & identity matrix & $\lambda$ & eigenvalue \\
\hline $\mathbf{K}$ & conductivity matrix & $\Delta t$ & time step \\
\hline & & $\omega_{b}$ & $\begin{array}{l}\text { blood perfusion } \\
\left(s^{-1}\right)\end{array}$ \\
\hline $\mathbf{M}_{L}$ & lumped capacity matrix & $\kappa$ & $\begin{array}{l}\text { thermal } \\
\text { conductivity } \\
\text { tensor }\left(\mathrm{Wm}^{-1}{ }^{0} \mathrm{C}^{-1}\right. \\
\text { ) }\end{array}$ \\
\hline$n q$ & number of equations & $\Psi$ & tissue damage \\
\hline$Q_{m}$ & metabolic heat of tissue $\left(\mathrm{Wm}^{-3}\right)$ & $\rho$ & density $\left(\mathrm{Kgm}^{-3}\right)$ \\
\hline$Q_{r}$ & spatial heating $\left(W^{-3}\right)$ & $\tau$ & $\begin{array}{l}\text { time (or dummy) } \\
\text { variable (s) }\end{array}$ \\
\hline$t$ & Time (s) & Superscripts & \\
\hline$t_{f}$ & time of analysis & $k$ & time index \\
\hline$T_{a}$ & arterial temperature & Subscripts & \\
\hline $\mathbf{T}(t)$ & time temperature vector & $b$ & blood \\
\hline
\end{tabular}




\section{Introduction}

In recent years, there has been an increasing motivation in the development of mathematical models that describe heat transfer in living tissue with blood perfusion. Among many mathematical models of bioheat transfer [1, 2], the Pennes' equation [3] is widely employed due to its simplicity and overall satisfactory representation of the physical phenomenon. Indeed, the Pennes' equation appears in a great number of bioheat applications such as hyperthermia, cryosurgery, hypothermia, thermography, skin burns, etc..

As the mathematical models and their applications in medical sciences are becoming more complex and multidisciplinary [1,2], numerical simulations are indispensible tools that partially replace laboratory testing, aiding in understanding the problem under consideration subjected to different inputs in an effective manner. In fact, over the years, the Pennes' bioheat transfer equation has been numerically solved by several numerical techniques such as the finite difference method [1, 4-6], finite element method $[1,7,8]$, boundary element method [9,10], meshless method [11,12] etc..

The objective of the present paper is to propose an improvement into the Explicit Green's approach method [13-17] and apply it to solve the Pennes' equation. As an application of the Pennes' equation, the paper focuses on the numerical simulation of skin burns [1, 6-8, 22] that can be used to predict injury depths caused by different external heat supplies applied at the skin surface. The generality and success of the ExGA method relies heavily on numerical Green's functions rather than analytical ones employed in other formulations. It is well-known that Green's function methodologies are very powerful tools due to the ability to solve the problem under consideration subjected to different boundary conditions and heat source terms [18-24]. For instance, one can quote the work of Deng and Liu [19] where analytical Green's functions for the Pennes' equation that satisfy the homogeneous boundary conditions of the same problem were employed. However, these analytical Green's functions, even though very important to derive benchmark solutions, are not feasible in practice due to the difficulty of finding analytical expressions for Green's functions with arbitrary geometries and/or material properties. In this sense, the ExGA uses numerical Green's functions that also satisfy homogeneous boundary conditions, giving rise to a general time-integral expression that can easily handle any kind of geometry and medium. Furthermore, 
unlike time-domain BEM formulations in which analytical free-space Green's functions are generally employed [25, 26], once Green's functions are computed in the ExGA method the solution is explicitly evaluated without the need of solving a system of equations.

The main contributions of the present paper are the proposed use and optimization of a new nonequal two time substeps procedure to compute Green's functions at the first time step, a detailed discussion on the computation of local numerical Green's functions and a convergence analysis of the technique. The FEM in conjunction with the explicit Euler scheme are employed to compute Green's functions, represented by the so-called Green's matrix that stores numerical nodal values of the Green's functions. The nonequal time substeps values are calculated such that a maximum stability region for the time-integral expression of the ExGA method is achieved. Because of the discretization adopted, the numerical Green's function due to a point source possesses a compact support with values different from zero only in a small region around the source point and, as a consequence, its computation can be carried out locally in a straightforward manner.

The structure of the paper is organized as follows: In section 2 the Pennes' bioheat transfer equation is briefly described and, in section 3, a brief background is given on the theoretical foundations of the time-integral expression regarding the ExGA method discretized in a FEM sense. Section 4 presents the time substeps procedure to compute the Green's matrix by means of the explicit Euler scheme and a discussion about the convolution integral. Next, in section 5, a detailed study of how to calculate the time substeps values by performing a stability and accuracy analysis is provided. In section 6, two numerical examples are presented in order to assess the capabilities and potentialities of the improved ExGA method, including a convergence study and a simulation of skin burns caused by a heated plate. Finally, conclusions on the proposed methodology are drawn in section 7.

\section{Model Equations}

In this work, biological systems, more specifically skin tissue subjected to external factors leading to burns, are modeled by means of the Pennes bioheat transfer equation. Let the biological system (skin tissue) occupy an open and bounded domain $\Omega \subset \mathbb{R}^{d}$ 
where $d$ is the order of space dimensions. The boundary of $\Omega$ is assumed to be sufficiently smooth and is denoted by $\Gamma=\partial \Omega$ with outward unit normal vector $\mathbf{n}$, $\bar{\Omega}=\Omega \bigcup \Gamma$ being the closure of $\Omega$. In this way, the Pennes' bioheat transfer model can be formulated as: find the tissue temperature field $T: \bar{\Omega} \times\left[0, t_{f}\right] \rightarrow \mathbb{R}$ such that $[1,2$, 3]:

$$
\begin{gathered}
\nabla \cdot(\mathbf{\kappa} \nabla T)+\omega_{b} \rho_{b} c_{b}\left(T_{a}-T\right)+Q_{m}+Q_{r}=\rho c \frac{\partial T}{\partial t} \text { in } \Omega \times\left(0, t_{f}\right] \\
T=\bar{T} \text { on } \Gamma_{D} \times\left(0, t_{f}\right] \\
\boldsymbol{\kappa} \nabla T \cdot \mathbf{n}=\bar{q} \text { on } \Gamma_{N} \times\left(0, t_{f}\right] \\
\kappa \nabla T \cdot \mathbf{n}=h\left(T_{\infty}-T\right) \text { on } \Gamma_{R} \times\left(0, t_{f}\right] \\
T=T_{0} \text { in } \Omega \text { at } t=0
\end{gathered}
$$

where the usual Dirichlet, Neumann and Robin (convective) type boundary conditions with their respective prescribed values and parameters are applied on $\Gamma_{D}, \Gamma_{N}$ and $\Gamma_{R}$, respectively, such that $\Gamma=\Gamma_{D} \cup \Gamma_{N} \cup \Gamma_{R}$ and $\Gamma_{D} \cap \Gamma_{N}=\Gamma_{D} \cap \Gamma_{R}=\Gamma_{N} \cap \Gamma_{R}=\varnothing$. In Eq.(1), $T_{a}$ stands for the arterial temperature which is treated as a constant, $Q_{m}: \Omega \rightarrow \mathbb{R}$ and $Q_{r}: \Omega \times\left[0, t_{f}\right] \rightarrow \mathbb{R}$ denote the metabolic heat generation and the supplied heat source, respectively. The tissue properties are: $\kappa: \bar{\Omega} \rightarrow \mathbb{R}^{d \times d}$ the thermal conductivity tensor, $\rho: \Omega \rightarrow \mathbb{R}$ the density and $c: \Omega \rightarrow \mathbb{R}$ the specific heat while the blood properties are: $\rho_{b}: \Omega \rightarrow \mathbb{R}, c_{b}: \Omega \rightarrow \mathbb{R}$ and $\omega_{b}: \Omega \rightarrow \mathbb{R}$ the blood perfusion. Finally, $T_{0}: \Omega \rightarrow \mathbb{R}$ is the initial temperature field.

Due to the lack of a precise knowledge of the initial temperature in the whole biological system adopted in the model, in many numerical simulations the initial temperature $T_{0}(\mathbf{x})$ is set as the solution of a previous steady-state problem governed by the equation below

$$
\nabla \cdot\left(\kappa \nabla T_{0}\right)+\omega_{b} \rho_{b} c_{b}\left(T_{a}-T_{0}\right)+Q_{m}=0 \text { in } \Omega
$$

subjected to appropriate boundary conditions. For instance, in skin burns simulations, the initial temperature may be accomplished by considering that the skin surface is 
under a convective boundary condition, a situation normally encountered previous to the heating that causes burns.

\section{Time Stepping Using the Green's Matrix}

The key feature of the ExGA method is the use of numerical Green's functions that satisfy homogeneous boundary conditions of the problem under consideration. Hence, according to Eqs. (1)-(5), the Green's function for the skin tissue can be derived from the following set of equations considering $\tau=0$ :

$$
\begin{gathered}
\nabla \cdot(\mathbf{\kappa} \nabla G(\mathbf{x}, \mathbf{y}, t))-\omega_{b} \rho_{b} c_{b} G(\mathbf{x}, \mathbf{y}, t)=\rho c \frac{\partial G(\mathbf{x}, \mathbf{y}, t)}{\partial t} \text {, in } \Omega \text { and } t>0 \\
G(\mathbf{x}, \mathbf{y}, t)=0, \text { on } \Gamma_{D} \text { and } t>0 \\
\mathbf{\kappa} \nabla G(\mathbf{x}, \mathbf{y}, t) \cdot \mathbf{n}=0 \text {, on } \Gamma_{N} \text { and } t>0 \\
\mathbf{\kappa} \nabla G(\mathbf{x}, \mathbf{y}, t) \cdot \mathbf{n}+h G(\mathbf{x}, \mathbf{y}, t)=0 \text {, on } \Gamma_{R} \text { and } t>0 \\
G(\mathbf{x}, \mathbf{y}, t)=\frac{\delta(\mathbf{x}-\mathbf{y})}{\rho c} \text { in } \Omega \text { and } t=0
\end{gathered}
$$

where $\mathbf{x} \in \mathbb{R}^{d}$ denotes the field point and $\mathbf{y} \in \mathbb{R}^{d}$ the source point. Notice that the Green's function calculation for $t>\tau=0$ can be carried out replacing the instantaneous heat point source by an equivalent initial condition expressed by Eq. (11) [18].

In this paper, the Green's function is numerically computed by the finite element method [27]. Thus, let $S_{t}^{h} \subset H_{0}^{1}(\Omega)$ and $V^{h} \subset H_{0}^{1}(\Omega)$ be the usual finite dimensional spaces of continuous piecewise polynomials concerned with trial and test functions on $\bar{\Omega}^{h}=\bigcup_{e} \bar{\Omega}_{e}$. The discrete counterpart of Eqs. (7)-(11) taking into account the semidiscretization Galerkin procedure can be stated as: for any $t \geq 0$ find $G^{h} \in S_{t}^{h}$ for $\mathbf{y}_{j}$ with $j \in \eta \backslash \eta_{D}$ such that $\forall w^{h} \in V^{h}$,

$$
\begin{gathered}
\left(w^{h}, \rho c \frac{\partial G^{h}}{\partial t}\right)+\left(\nabla w^{h}, \mathbf{\kappa} \nabla G^{h}\right)+\left(w^{h}, \omega_{b} \rho_{b} c_{b} G^{h}\right)+\left(w^{h}, h G^{h}\right)_{\Gamma_{R}}=0 \\
\left(w^{h}, \rho c G^{h}\right)=\left(w^{h}, \delta\left(\mathbf{x}-\mathbf{y}_{j}\right)\right)
\end{gathered}
$$


where the standard notation for the $L_{2}$ inner product has been used. In addition, $\eta$ is the total number of nodes in the mesh with $\eta_{D}$ being its subset of nodes belonging to $\Gamma_{D}$.

Defining $G^{h}\left(\mathbf{x}, \mathbf{y}_{j}, t\right)=\sum_{i \in \eta \backslash \eta_{D}} N_{i}(\mathbf{x}) G_{i}\left(\mathbf{y}_{j}, t\right)$ and $w^{h}(\mathbf{x})=\sum_{l \in \eta \backslash \eta_{D}} N_{l}(\mathbf{x}) w_{l} \quad$ in which $N(\mathbf{x})$ are the global finite element shape functions, and after taking into account all source points $\mathbf{y}_{j}$, Eqs. (12)-(13) can be rewritten in matrix notation as: find $\mathbf{G}: t \geq 0 \rightarrow \mathbb{R}^{n q \times n q}$ such that:

$$
\begin{gathered}
\mathbf{M}_{L} \dot{\mathbf{G}}(t)+\mathbf{K G}(t)=\mathbf{0} \\
\mathbf{G}(0)=\mathbf{M}_{L}^{-1}
\end{gathered}
$$

where $\mathbf{M}_{L} \in \mathbb{R}^{n q \times n q}, \mathbf{K} \in \mathbb{R}^{n q \times n q}$ denote, respectively, the capacitance and conductivity matrices [17] with $n q$ being the number of equations originated from unknown nodal values associated with the subset $\eta \backslash \eta_{D}$. The Green's matrix, with entries expressed by $G_{i j}(t) \equiv G^{h}\left(\mathbf{x}_{i}, \mathbf{y}_{j}, t\right)$, actually represents Green's functions nodal values of the discrete system. An advantage of computing the Green's function numerically by the FEM is that the compatibility conditions between layers, for non-homogeneous media, are taken into account directly into the FEM formulation. Thus, unlike other methods such as the BEM, it is not necessary to adopt, for instance, the subregion technique that makes use of the compatibility conditions explicitly in its formulation due to the use of a fundamental solution of homogeneous media [26]. In the foregoing equations a lumped (diagonal) capacitance matrix originated from a diagonalization procedure such as the row sum technique $[27,28]$ is employed in order to render a more computational effective methodology since its inversion is readily obtained.

To establish the solution of Eqs. (1)-(5) by means of the ExGA method, the governing equation is multiplied by the Green's function discussed above and integrated over the space-time domain $\Omega \times\left(t_{0}, t_{f}\right]$. Afterwards, following some usual procedures of standard time domain boundary integral equations and performing a spatial discretization similar to that of the FEM, the problem becomes: find the temperature vector $\mathbf{T}:\left(t_{0}, t_{f}\right] \rightarrow \mathbb{R}^{n q}$ such that $[14,17]$ : 


$$
\mathbf{T}(t)=\mathbf{G}\left(t-t_{0}\right) \mathbf{M}_{L} \mathbf{T}\left(t_{0}\right)+\int_{t_{0}}^{t} \mathbf{G}(t-\tau) \mathbf{F}(\tau) d \tau, \quad t \in\left[t_{0}, t_{f}\right]
$$

where $\mathbf{F}:\left(t_{0}, t_{f}\right) \rightarrow \mathbb{R}^{n q}$ stands for the usual heat load vector, accounting for boundary conditions and heat sources. For more details regarding the derivation of Eq. (16), the reader is referred to Loureiro et al. [14].

To take advantage of computing the Green's matrix only at a given time step and evaluating the convolution integral only once, the temperature vector computation in Eq. (16) is carried out by a step-by-step time-marching process. In this way, the total time interval $\left[t_{0}, t_{f}\right]$ is split into $N$ equal subintervals $\left[t_{k}, t_{k+1}\right]$ with $0=t_{0}<t_{1}<\cdots<t_{N}=t_{f}$ and $t_{k+1}-t_{k}=\Delta t=t_{f} / N$. Finally, after taking into consideration the Green's function translation property, the following recursive expression arises:

$$
\mathbf{T}^{k+1}=\mathbf{G}(\Delta t) \mathbf{M}_{L} \mathbf{T}^{k}+\int_{0}^{\Delta t} \mathbf{G}(\Delta t-\tau) \mathbf{F}\left(t_{k}+\tau\right) d \tau, k=0, \ldots, N-1
$$

in which subscript $k$ indicates the time instant, e.g., $\mathbf{T}^{k} \equiv \mathbf{T}\left(t_{k}\right)$.

\section{Green's Matrix Computation}

In this section, a discussion concerning the computation of Green's functions or, in other words, the Green's matrix at the first time instant by a two time substeps methodology is presented. Because of the discretization employed, the Green's function possesses compact support and computational advantages can be taken into account from this fact in order to establish a more effective algorithm.

\subsection{A two time substeps procedure}

It is well known that the explicit Euler time integration method is widely adopted to solve systems of parabolic ordinary differential equations that arise in many finite element simulations $[27,28]$. However, the major drawback of this explicit 
method is its severe stability constraint. Motivated by the flexibility offered by the ExGA method, the Green's matrix at time instant $t=\Delta t$ is computed by the Euler method in conjunction with a two time substeps procedure, aiming to relax the stability constraint. Thus, applying the explicit Euler method to Eqs. (14)-(15), one obtains:

$$
\mathbf{G}\left(t_{i}\right)=\left(\mathbf{I}-\alpha_{i} \Delta t \mathbf{M}_{L}^{-1} \mathbf{K}\right) \mathbf{G}\left(t_{i-1}\right), i=1,2
$$

where $t_{i}=\sum_{j=1}^{i} \alpha_{j} \Delta t$ and $\mathbf{I} \in \mathbb{R}^{n q \times n q}$ is the identity matrix.

Figure 1 illustrates the time subteps procedure which are employed only once, for the first time step. The main goal now is to optimize the values of $\alpha_{1} \Delta t$ and $\alpha_{2} \Delta t$ such that $\alpha_{1} \Delta t+\alpha_{2} \Delta t=\Delta t$ (or $\alpha_{1}+\alpha_{2}=1$ ), focusing on increasing the stability region as much as possible without reducing accuracy. This follows a different approach to that proposed by Loureiro and Mansur [16] where the focus was on an increase in the order of accuracy. The optimal values for $\alpha_{1}$ and $\alpha_{2}$ are discussed in detail in the next section.

\subsection{Convolution integral}

Once the Green's matrix has been computed by the Euler method, one can proceed with the approximation of the convolution integral in order to initialize the time marching process for the temperature vector calculation given by Eq. (17). Actually, the convolution integral is dealt with analytically considering a type of time variation for the heat load vector and the only error is due to the use of an approximate Green's matrix given by Eq. (18) as shown in appendix A. As presented in Appendix A, the convolution integral reads:

$$
\int_{0}^{\Delta t} \mathbf{G}(\Delta t-\tau) \mathbf{F}\left(t_{k}+\tau\right) d \tau \approx \mathbf{G}(0)\left(\mathbf{F}^{k} \alpha_{1}^{2} \Delta t+\mathbf{F}^{k+1} \alpha_{1} \alpha_{2} \Delta t\right)+\mathbf{G}\left(\alpha_{1} \Delta t\right) \mathbf{F}^{k} \alpha_{2} \Delta t
$$

The above expression assumes that the heat load vector varies linearly for each time subinterval $\left[t_{k}, t_{k+1}\right]$. This assumption is normally used in several time integration methods $[27,28]$ and is sufficient to yield accurate results for a small enough time step 
when compared to the time range of the analysis. However, other types of time shape functions for the heat load vector can be considered as well.

\subsection{Local Green's functions}

Due to the discretization adopted in both time and space, the Green's function nodal values obtained from Eq. (18) correspond to a column of the Green's matrix, and admit compact support. This means that the Green's function associated to a specific source point $\mathbf{y}_{j}, j \in \eta \backslash \eta_{D}$ needs to be computed only in a small portion of the mesh surrounding the source point $\mathbf{y}_{j}$ with its respective discrete local subdomain (submesh), namely, $\Omega_{G_{j}}^{h} \subseteq \Omega^{h}, j \in \eta \backslash \eta_{D}$. Figure 2 displays non-null nodal values of the Green's function in $\Omega_{G_{j}}^{h}$ by employing, for instance, quadrilateral elements that are adopted here.

Further considerations about the Green's functions computation can be taken into account by invoking the reciprocity relation. The reciprocity relation states that the Green's function is symmetric when the source and field points are interchanged independent of the position of these points or, in other words, $G(\mathbf{x}, \mathbf{y}, t-\tau)=G(\mathbf{y}, \mathbf{x}, t-\tau)$. It is readily seen from Eq. (18) that this property also holds for the Green's matrix implying in a symmetric matrix and, therefore, only nodal Green's function values for indices say $i \leq j$ must be computed, reducing the computational cost by half.

In some simulations, it is quite common to adopt uniform elements at least in some portion of the finite element mesh and a piecewise homogeneous material where material properties are constant in each subdomain. If the local Green's function subdomain $\bar{\Omega}_{G_{j}}^{h}$ is generated only by regular elements with the same kind of material, it is very useful to compute the Green's function considering only one-quarter of the model as depicted in Figure 2(b). This not only decreases the computational cost but also indicates that the Green's function can be reused for other equal local subdomains $\bar{\Omega}_{G_{j}}^{h}$ which is more likely to occur when a fine mesh is employed, increasing drastically the computational efficiency. Notice that the Dirichlet type boundary condition is adopted to truncate the local mesh inside the original mesh, i.e. nodal values from the local boundary to the rest of the mesh are null. On the other hand, if the local boundary 
$\Gamma_{G_{j}}^{h}$ intercepts the original boundary, the last local boundary with its respective boundary condition must be considered. Although not addressed in this work, it is important to stress that the proposed formulation is highly parallelizable since Green's functions with their corresponding discrete local subdomains are computed independently and only matrix-vector operations are required in the recurrence relation (17) for the temperature vector.

\section{Optimal Values for the Time Substeps}

The parameters $\alpha_{1}$ and $\alpha_{2}$ which define the substeps in the proposed time-stepping scheme play a central role in the time convergence. It is well-known from the Lax equivalence theorem that a time-stepping technique for linear problems is convergent if and only if it is stable and consistent. Following [27, 28], this section presents a detailed study on the influence of these parameters into the stability and accuracy in time, taking into account a decoupled procedure for the system of equations.

\subsection{Decoupled equations}

Let $\left(\lambda_{1}, \boldsymbol{\varphi}_{1}\right),\left(\lambda_{2}, \boldsymbol{\varphi}_{2}\right), \ldots,\left(\lambda_{n q}, \boldsymbol{\varphi}_{n q}\right)$ be the eigenvalues and their corresponding eigenvectors associated with the eigenvalue problem [27, 28]

$$
\left(\mathbf{M}_{L}^{-1} \mathbf{K}-\lambda_{l} \mathbf{I}\right) \boldsymbol{\varphi}_{i}=\mathbf{0}, i \in\{1, \ldots, n q\}
$$

for which $0 \leq \lambda_{1} \leq \ldots \leq \lambda_{n q}$. The eigenvectors satisfy the orthonormality relations $\boldsymbol{\varphi}_{i}^{T} \mathbf{M}_{L} \boldsymbol{\varphi}_{i}=\delta_{i j}$ and $\boldsymbol{\varphi}_{i}^{T} \mathbf{K} \boldsymbol{\varphi}_{i}=\lambda_{i} \delta_{i j}$. Then, as $\boldsymbol{\varphi}_{i}, i=1, \ldots, n q$ form a basis for $\mathbb{R}^{n q}$, one can write $\mathbf{T}(t)=\sum_{i=1}^{n q} \boldsymbol{\varphi}_{i} T_{i}(t)$. Finally, substituting the aforementioned expression for the temperature vector into Eq. (17), taking into consideration Eq. (18) and Eq. (19), and with the aid of the orthonormality relations, the following decoupled scalar recursive expressions are obtained:

$$
T_{i}^{k+1}=g_{i}^{h}\left(\lambda_{i} \Delta t, \alpha_{1}\right) T_{i}^{k}+L_{i}^{h}, i=1, \ldots, n q
$$


where $g_{i}^{h}\left(\lambda_{i} \Delta t, \alpha_{1}\right)$ can be interpreted as the approximate Green's function or the socalled amplification factor, and $L_{i}^{h}$ is the approximate convolution integral or the load operator. Owing to the relation $\alpha_{2}=1-\alpha_{1}$ their expressions are written as

$$
\begin{gathered}
g_{i}^{h}\left(\lambda_{i} \Delta t, \alpha_{1}\right)=\left(1-\alpha_{1} \Delta t \lambda_{i}\right)\left(1-\left(1-\alpha_{1}\right) \Delta t \lambda_{i}\right) \\
L_{i}^{h}=\alpha_{1}^{2} \Delta t r_{i}^{k}+\alpha_{1} \alpha_{2} \Delta t r_{i}^{k+1}+\left(1-\alpha_{1} \Delta t \lambda_{i}\right) \alpha_{2} \Delta t r_{i}^{k}
\end{gathered}
$$

in which $r_{i}(t)=\boldsymbol{\varphi}_{i}^{T} \mathbf{F}(t)$. Conversely, analytical counterparts of the Green's function and the load operator for a SDOF system are $g_{i}\left(\lambda_{i} \Delta t\right)=e^{-\lambda_{i} \Delta t}$ and $L_{i}=\int_{0}^{\Delta t} e^{-\lambda_{i}(\Delta t-\tau)} r_{i}\left(t_{k}+\tau\right) d \tau$, respectively.

The decoupled procedure allows, without loss of generality, the analysis of the stability and accuracy of a given time-stepping scheme in a straightforward manner, and conclusions drawn for decoupled equations remain valid for the original system of coupled equations.

\subsection{Time stability region}

It is easy to see from the analytical Green's function $g_{i}\left(\lambda_{i} \Delta t\right)=e^{-\lambda_{i} \Delta t}$ that the recursive expression (21) is unconditionally stable only if the condition $\left|g_{i}^{h}\left(\lambda_{i} \Delta t, \alpha_{1}\right)\right| \leq 1, \forall i \in\{1, \ldots, n q\}$ holds; otherwise, the time-stepping scheme is said to be conditionally stable. Since the approximate Green's function also depends on the parameters $\alpha_{1}$ and $\alpha_{2}=1-\alpha_{1}$, these parameters are optimized to ensure a maximum stability region. In other words, we seek the value of $\alpha_{1}$ such that $\left|g_{i}^{h}\left(\lambda_{i} \Delta t, \alpha_{1}\right)\right| \leq 1$ for the largest range of $\lambda_{i} \Delta t$.

Figure 3 shows the $3 \mathrm{D}$ and stability region plots of $g_{i}^{h}\left(\lambda_{i} \Delta t, \alpha_{1}\right)$ in terms of $\lambda_{i} \Delta t$ considering $\alpha_{1} \in[0,1]$. Like the standard explicit Euler method, it is readily observed from Figure 3 that the proposed time-stepping is only conditionally stable. Because the stability is greatly affected by the parameter $\alpha_{1}$, the goal is to find an 
optimal value of $\alpha_{1}$ that yields the largest stability region. This optimal value is illustrated by a line in Figure 3(b), and it is determined by solving the equations

$$
g_{i}^{h}\left(\lambda_{i} \Delta t, \alpha_{1}\right)=-1 \& \& \frac{\partial g_{i}^{h}\left(\lambda_{i} \Delta t, \alpha_{1}\right)}{\partial\left(\lambda_{i} \Delta t\right)}=0
$$

which leads to $\alpha_{1}=\frac{2-\sqrt{2}}{4}$. In fact, the optimal value is that for which the vertical line in Figure 3(b) is tangent to the point $\left(\alpha_{1}, \lambda_{i} \Delta t\right)=\left(\frac{2-\sqrt{2}}{4}, 4\right)$. In addition, three curves for $g_{i}^{h}\left(\lambda_{i} \Delta t, \alpha_{1}\right)$ considering different values of $\alpha_{1}$ are plotted in Figure 4 and some features about the two time substeps can also be outlined from this figure and Figure 3, namely: i) if $\alpha_{1}=1$ no substep is established and the standard Euler stability constraint given by $\lambda_{i} \Delta t \leq 2$ is obtained; ii) for $\alpha_{1}=1 / 2$, i.e. equal substeps [13, 15], the stability constraint is $\lambda_{i} \Delta t \leq 4$ and iii) for the optimal $\alpha_{1}$, the stability constraint is increased to $\lambda_{i} \Delta t \leq 8$. In this sense, the two time substeps procedure with $\alpha_{1}=\frac{2-\sqrt{2}}{4}$ is capable of enlarging the critical time step by a factor of 4 in comparison with the standard Euler method and a factor of 2 when compared to the equal substeps. Hence, a new timestepping scheme with stability improvement is established.

\subsection{Order of accuracy in time}

The final task to achieve a convergent solution is concerned with the order of accuracy of the time integration method. The order of accuracy can be analyzed by comparing the Taylor series of the analytical Green's function $g_{i}\left(\lambda_{i} \Delta t\right)$ with that of $g_{i}^{h}\left(\lambda_{i} \Delta t, \alpha_{1}\right)$ [27, 28]. The Taylor series $g_{i}\left(\lambda_{i} \Delta t\right)$ is written as

$$
g_{i}\left(\lambda_{i} \Delta t\right)=e^{-\lambda_{i} \Delta t}=1-\lambda_{i} \Delta t+\frac{1}{2} \lambda_{i}^{2} \Delta t^{2}-\ldots+\frac{1}{n !} \lambda_{i}^{n} \Delta t^{n}-\ldots=\sum_{n=0}^{\infty}(-1)^{n} \frac{\lambda^{n} \Delta t^{n}}{n !}
$$

while the expansion for $g_{i}^{h}\left(\lambda_{i} \Delta t, \alpha_{1}\right)$ is given by 


$$
g_{i}^{h}\left(\lambda_{i} \Delta t, \alpha_{1}\right)=1-\lambda_{i} \Delta t+\left(\alpha_{1}-\alpha_{1}^{2}\right) \lambda_{i}^{2} \Delta t^{2}
$$

By definition [27, 28], a time integration scheme is said to be of order $p$ if all terms of the Taylor series regarding the analytical Green's function up to $\left(\lambda_{i} \Delta t\right)^{p}$ match exactly those of the numerical Green's function. In other words, the local truncation error for the proposed formulation can be defined as $\left|g_{i}\left(\lambda_{i} \Delta t\right)-g_{i}^{h}\left(\lambda_{i} \Delta t, \alpha_{1}\right)\right| \leq c\left(\alpha_{1}\right)\left(\lambda_{i} \Delta t\right)^{2}$, where $c\left(\alpha_{1}\right)=1 / 2-\alpha_{1}+\alpha_{1}^{2} \quad$ is a constant dependent of $\alpha_{1}$. Clearly, the proposed time-stepping method is first order accurate and, consequently, it is consistent. Furthermore, from a theoretical point of view, when $c\left(\alpha_{1}\right)$ approaches to zero a better accuracy is obtained. A few comments about the constant $c\left(\alpha_{1}\right)$ whose graph is plotted in Figure 5 can be outlined, namely: i) the best accuracy is achieved when $\alpha_{1}=1 / 2$, i.e., when equal time substeps are employed; ii) when $\alpha_{1}=1$ (Euler method) one has $c\left(\alpha_{1}\right)=0.5$, whereas when $\alpha_{1}=\frac{2-\sqrt{2}}{4}$ gives $c\left(\alpha_{1}\right)=0.375$ and, therefore, the proposed method is slightly more accurate than the explicit Euler one; iii) there is no $\alpha_{1} \in \mathbb{R}$ such that $c\left(\alpha_{1}\right)=0$, i.e., a second order accurate method, and one needs to rely on the complex plane to increase the order of accuracy resulting in $\alpha_{1} \in \mathbb{C}$, as shown by Loureiro and Mansur [16]. Although equal substeps yield the best accuracy, the maximum stability generated by $\alpha_{1}=\frac{2-\sqrt{2}}{4}$ is much more important than the accuracy when dealing with finite element simulations containing a large number of equations since the time step is constrained by the largest eingenvalue which, in general, is inaccurately computed by the FEM and not very important to the problem analysis.

\section{Numerical Aspects and Applications}

In the present section two examples are considered. In the first one, the convergence of the proposed formulation according to the time substeps selection is studied by considering a two-degree-of-freedom model. In the second example, an application 
related to skin burn depth simulations is analyzed with the goal of describing the main features of the proposed method.

\subsection{Convergence study}

In order to show that the order of accuracy demonstrated previously for the decoupled equations remains valid for the system of coupled equations originated from the FEM semi-discretization applied to the Pennes' equation, the following two-degree-offreedom model is considered:

$$
\left[\begin{array}{ll}
4 & 0 \\
0 & 3
\end{array}\right]\left\{\begin{array}{c}
\dot{T}_{1}(t) \\
\dot{T}_{2}(t)
\end{array}\right\}+\left[\begin{array}{cc}
5 & -3 \\
-3 & 3
\end{array}\right]\left\{\begin{array}{c}
T_{1}(t) \\
T_{2}(t)
\end{array}\right\}=\left\{\begin{array}{c}
1-t \\
0
\end{array}\right\}
$$

with

$$
\left[\begin{array}{l}
T_{1}(0) \\
T_{2}(0)
\end{array}\right]=\left[\begin{array}{l}
1 \\
0
\end{array}\right]
$$

The convergence rate or order of accuracy of the proposed time-stepping technique is numerically estimated by computing the solution at a specific time instant considering different time steps. The error is calculated by the following expression:

$$
e(t)=\frac{\left\|\mathbf{T}^{\text {num }}(t)-\mathbf{T}^{a n}(t)\right\|_{2}}{\left\|\mathbf{T}^{a n}(t)\right\|_{2}}
$$

where $\mathbf{T}^{a n}(t), \quad \mathbf{T}^{\text {num }}(t)$ stand for analytical and numerical solution vectors, respectively. The expression for the analytical solution reads

$$
\left\{\begin{array}{l}
T_{1}(t) \\
T_{2}(t)
\end{array}\right\}=\left\{\begin{array}{c}
\frac{1}{28}\left(63+13 e^{-2 t}-48 e^{-t / 4}-14 t\right) \\
\frac{1}{28}\left(77-13 e^{-2 t}-64 e^{-t / 4}-14 t\right)
\end{array}\right\}
$$

Figure 6 shows the convergence rate at the time instant $t_{r}=1$ for the standard explicit Euler method and the ExGA method. From this figure, it can be concluded that the ExGA method has the same order of accuracy as the Euler scheme (first order accurate). However, it is slightly more accurate (smaller error) than the Euler scheme 
with the ExGA method with $\alpha_{1}=1 / 2$ (equal substeps) being the most accurate as stated previously in the theoretical discussion.

\subsection{Skin burn simulation}

As an application of a bioheat transfer model, the tissue temperature and its effects into tissue damage when the skin surface is exposed to a heated plate are studied. Numerical simulations can be effectively used to better understand burn injuries caused by different heat-supply situations and, as a consequence, aiding in the investigation of more appropriate postburn cooling therapies and the development of clinical protocols $[5,7,8,6]$. A two dimensional model is considered as depicted in Figure 7 where the tissue is formed by four layers, namely, Epidermis, Dermis, Subcutaneous and Muscle. On the skin surface, the heated plate is applied at a portion of the boundary at a prescribed temperature while the remaining boundary is subjected to a convection boundary condition. The core boundary is at $37^{\circ} \mathrm{C}$ while the upper and lower ones are assumed to be insulated. The thickness and material properties of the layers are given in Table 1 and the metabolic heat generation and the arterial temperature are set to be $Q_{m}=420 \mathrm{~W} / \mathrm{m}^{3}$ and $T_{a}=37^{\circ} \mathrm{C}$, respectively. Due to symmetry, only half of the domain is considered and a total of 12745 bilinear elements are adopted for the spatial discretization.

Since the tissue is subjected to a convection boundary condition at the skin surface before exposure to the heated plate, the initial temperature of the model is calculated by solving the steady state version of the bioheat transfer equation. Due to the boundary conditions the problem is actually reduced to a $1 \mathrm{D}$ model as shown in Figure 8, considering an ambient temperature of $T_{\infty}=20^{\circ} \mathrm{C}$ and a convective heat transfer coefficient of $h=10 \mathrm{~W} / \mathrm{m}^{2}{ }^{0} \mathrm{C}$. As expected, temperatures near the skin surface are below that of the body core, which is around $37^{\circ} \mathrm{C}$.

In skin burn simulations, it is very important to classify the degree of tissue burn. To accomplish this, the thermal tissue damage (or injury) region $\Psi: \bar{\Omega} \times[0, t] \rightarrow \mathbb{R}$ is measured by the Arrhenius model [1], which in its integral form is given by 


$$
\Psi=\int_{0}^{t} \xi e^{-\frac{\Delta E}{R(T+273)}} d \tau
$$

where $R$ is the universal gas constant and $T: \bar{\Omega} \times[0, t] \rightarrow \mathbb{R}$ is the local temperature. Furthermore, $\Delta E$ stands for the free energy barrier to molecular denaturation while $\xi$ is a constant determined by the tissue properties. The burn injury is classified according to the values of $\Psi$ with $0.53 \leq \Psi<1,1 \leq \Psi<10^{4}$ and $\Psi \geq 10^{4}$ being first, second and third degree burns, respectively.

Once the initial temperature is known the analysis can proceed. In the present simulation, a hot plate at $60^{\circ} \mathrm{C}$ is suddenly applied at the skin surface and the exposure is maintained for 294s, which is mathematically represented by the prescribed temperature function $\bar{T}=60(H(t)-H(t-294))$ with $H(\bullet)$ being the generalized Heaviside function. Afterwards, the plate is removed and the whole skin surface is again subjected to the natural convective boundary condition described above, which will now have a cooling effect. The objective is to analyze both temperature variations inside the tissue and the tissue thermal damage during the exposure and after the hot plate has been removed. The parameters used in Eq. (31) are $\xi=3.1 \times 10^{98} 1 / \mathrm{s}$ and $\Delta E=6.28 \times 10^{8} \mathrm{~J} / \mathrm{kmol}[7]$.

The solution is carried out considering the standard explicit Euler scheme, which is used as a reference solution, and the proposed ExGA method. The time step length is selected owing to the stability constraint of each method. The maximum eigenvalue for the mesh adopted in the problem is $\lambda_{\max }=135.538$. This means that $\Delta t \leq 0.014756$ for the Euler scheme and $\Delta t \leq 0.05902$ for the ExGA method to guarantee stability of the numerical results, so that $\Delta t=0.014 \mathrm{~s}$ is employed here for the Euler scheme and $\Delta t=0.056 \mathrm{~s}$ for the ExGA method. In practice, the maximum eigenvalue may be readily estimated through a much less computationally demanding procedure, owing to the fact that $\lambda_{\max } \leq \max _{e} \lambda_{e}$ in which $\lambda_{e}$ are element eigenvalues. It is important to stress that the time step length concerning the ExGA method is four times greater than that of the Euler scheme; besides, the former is more accurate than the latter as discussed previously. These are major advantages of the proposed formulation over the Euler scheme since a reduction of $75 \%$ for the number of time steps is achieved without 
deteriorating the solution. Moreover, concerning local Green's functions, one can observe that a regular mesh is employed in the inner layer (Muscle) and, thus, local Green's functions that share the same submesh (i.e., $\bar{\Omega}_{G_{j}}^{h}$ ) with the same material properties when source nodal points are placed at the inner layer are equal, reducing drastically the Green's functions calculation. Hence, in this case, the computation of the local Green's function at nodal points is performed just once with the symmetry shown in Figure 2(b) being taken into account to further reduce the computational cost. Therefore, this example reveals that in a great deal of cases one can take advantage of the mesh pattern in order to reduce the computational cost of evaluating local Green's functions.

Some results of the numerical simulation are presented in Figure 9-11, and it can be observed the results provided by the ExGA method are in a very good agreement with those of the Euler scheme. Before discussing the results, it is worth mentioning that the tissue thermal damage starts to occur at $43{ }^{\circ} \mathrm{C}$. Figure 9(a) shows the temperature-time histories at three distinct points with the first one located at the interface between Epidermis and the Dermis, the second at the middle of the Dermis layer and the third at the middle of the Subcutaneous layer, with all of them at $y=0$. As expected, temperatures are higher near the skin surface where we can see that the temperature is clearly above $43^{\circ} \mathrm{C}$ at points in the Epidermis and Dermis. After the hot plate is removed the temperature starts to decrease due to the convective cooling. In Figure 9(b) the temperature distribution at time instants $t=294 \mathrm{~s}$ (just before the plate has been removed) ant $t=588 \mathrm{~s}$ considering $y=0$ are plotted, and it is seen that as the position $y$ increases the temperature values decrease since the hot plate is applied only at one portion of the boundary. An interesting fact to be observed is that the thermal injury continues to occur at inner layers even after the hot plate removal. This is more clearly observed in Figure 10 for the tissue thermal damage where its values at time $t=588 \mathrm{~s}$ are higher than those at $t=294 \mathrm{~s}$ for inner layers (Subcutaneous and Muscle). Moreover, according to this figure, a second degree burn occurs at the Epidermis, Dermis and at the beginning of the Subcutaneous layers. Finally, Figure 11 shows the temperature field at $t=294 \mathrm{~s}$ and $t=588 \mathrm{~s}$ where once again we can see the temperature increase in inner layers after the hot plate is removed. 


\section{Conclusions}

In this work, an improvement into the Explicit Green's Approach (ExGA) formulation regarding stability has been presented for the Pennes bioheat transfer equation. The ExGA method is divided into two phases, namely, computation of the Green's functions represented by the Green's matrix and solution of the problem, i.e., finding the temperature vector through a recursive time relation that depends on the Green's matrix.

The new feature of the proposed formulation is to compute the Green's matrix at $t=\Delta t$ by splitting this time step into two nonequal time substeps, with their values being determined such that a maximum stability region is achieved for the temperature recursive relation. The explicit Euler scheme is chosen to compute the Green's matrix since it is widely used and very easy to program. After finding optimal values for the time substeps the following advantages over the standard Euler scheme arise: $i)$ the stability constraint is increased by a factor of four; ii) the first-order accuracy is maintained but with a lower error; iii) the temperature vector time advancement is carried out with a time step length $\Delta t$ without any substeps procedure; and iv) Green's functions can be saved and reused when multiple analyses with different boundary and source terms are considered. Thus, the proposed methodology allows the use of larger time steps without decreasing the accuracy, rendering a very efficient and accurate time stepping scheme.

The idea of local numerical Green's functions, where their values are non-zero only for a few nodal points around the source point, has also been presented. The objective is to compute the nodal Green's functions at a low cost, taking into account some properties of the discrete local subdomain as well as the symmetry of the local mesh. This is illustrated by the skin burn numerical example where regions with the same local mesh have the same nodal numerical Green's function and, therefore, their computation needs to be performed just once. Hence, the proposed formulation is a very attractive time-stepping technique based on local numerical Green's functions that can be easily extended to other bioheat mathematical models governed by parabolic equations. 


\section{Appendix A}

A discussion on the analytical evaluation of the convolution integral matrix that appears in Eq. (17) is provided here. The convolution integral is written as:

$$
\mathbf{I}_{c}=\int_{0}^{\Delta t} \mathbf{G}(\Delta t-\tau) \mathbf{F}\left(t_{k}+\tau\right) d \tau
$$

From the theory of ordinary differential equations the analytical Green's matrix governed by Eqs. (14)-(15) reads $\mathbf{G}_{A}(t)=e^{-\mathbf{M}_{L}^{-1} \mathbf{K} t} \mathbf{M}_{L}^{-1}$. Finally, assuming for instance that the heat load has a linear time variation, i.e., $\mathbf{F}\left(t_{k}+\tau\right)=\mathbf{F}^{k}+\left(\mathbf{F}^{k+1}-\mathbf{F}^{k}\right) \tau / \Delta t, \tau \in[0, \Delta t]$, the integral (A1) can be analytically evaluated, giving rise to

$$
\mathbf{I}_{c}=\int_{0}^{\Delta t} e^{-\mathbf{M}_{L}^{-1} \mathbf{K}(\Delta t-\tau)} \mathbf{M}_{L}^{-1}\left(\mathbf{F}^{k}+\left(\mathbf{F}^{k+1}-\mathbf{F}^{k}\right) \tau / \Delta t\right) d \tau=-\mathbf{G}_{A}(\Delta t) \mathbf{M}_{L} \boldsymbol{\psi}_{1}+\boldsymbol{\psi}_{1}+\boldsymbol{\psi}_{0} \Delta t \text { (A2) }
$$

where vectors $\boldsymbol{\psi}_{0}$ and $\boldsymbol{\psi}_{1}$ are defined as

$$
\begin{gathered}
\boldsymbol{\Psi}_{0}=\mathbf{K}^{-\mathbf{1}} \frac{\left(\mathbf{F}^{k+1}-\mathbf{F}^{k}\right)}{\Delta t} \\
\boldsymbol{\Psi}_{1}=\mathbf{K}^{-\mathbf{1}}\left(\mathbf{F}^{k}-\mathbf{M}_{L} \boldsymbol{\Psi}_{0}\right)
\end{gathered}
$$

This is indeed the so-called particular or steady-state solution studied by Loureiro and Mansur [15, 16], for which the time shape function concerned with the heat load vector is represented exactly into the final solution. The key feature now is to replace the analytical Green's matrix by its numerical counterpart, i.e., $\mathbf{G}_{A}(\Delta t) \approx \mathbf{G}(\Delta t)$. Hence, substituting the numerical Green's matrix given by Eq. (18) into Eq. (A2) and after some algebraic manipulations, one finally obtains:

$$
\int_{0}^{\Delta t} \mathbf{G}(\Delta t-\tau) \mathbf{F}\left(t_{k}+\tau\right) d \tau \approx \mathbf{G}(0)\left(\alpha_{1}^{2} \Delta t \mathbf{F}^{k}+\alpha_{1} \alpha_{2} \Delta t \mathbf{F}^{k+1}\right)+\mathbf{G}\left(\alpha_{1} \Delta t\right) \mathbf{F}^{k} \alpha_{2} \Delta t
$$


It is worth mentioning that the approximation of Eq. (A5) is not due to a quadrature formula but rather due to the use of an approximate Green's matrix. Thus, it can be inferred that the error for the temperature vector in the time marching process is originated only from the Green's matrix approximation.

\section{Acknowledgements}

The financial support provided by FAPEMIG, CNPQ and UFJF is greatly acknowledged.

\section{References}

1. B. Rubinsky, Numerical bioheat transfer, in Minkowycz W.J., Sparrow E.M., Murthy J.Y. (eds.), Handbook of Numerical Heat Transfer, 2nd ed., John Wiley and Sons, New Jersey, 2006, pp. 851-893.

2. K. Khanafer, K. Vafai, "Synthesis of Mathematical Models Representing Bioheat Transport", in: Minkowycz W.J., Sparrow E.M. (eds.), Advances in Numerical Heat Transfer, Vol. 3, Taylor \& Francis, New York, 2009, pp. 1-28.

3. H.H. Pennes, Analysis of tissue and arterial temperatures in the resting human forearm, J. Appl. Physiol. 1 (1948) 93-122.

4. W. Dai, H. Yu, R. Nassar, A fourth-order compact finite difference scheme for solving a 1-D Pennes bioheat transfer equation in a triple-layered skin structure, Numer. Heat Tran. Part B 46 (2004) 447-461.

5. S.C. Jiang, N. Ma, H.J. Li, X.X. Zhang, Effects of thermal properties and geometrical dimensions on skin burn injuries, Burns 28 (2002) 713-717.

6. N.N. Johnson, J.P. Abraham, Z.I. Helgeson, W.J. Minkowycz, E.M. Sparrow, An archive of skin-layer thicknesses and properties and calculations of scald burns with comparisons to experimental observations, J Therm Sci Eng Appl 3 (2011) Paper no. 011003.

7. D.A. Torvi, J.D. Dale, A finite element model of skin subjected to a flash fire, ASME J. Biomech. Eng. 116 (1994) 250-255.

8. E.Y.K. Ng, L.T. Chua, Comparison of one- and two-dimensional programmers for predicting the state of skin burns, Burns, 28 (2002), 27-34. 
9. C.L. Chan, Boundary element method analysis for the bioheat transfer equation, ASME J. Biomech. Eng. 114 (1992) 358-65.

10. J. Zhou, Y. Zhang, J.K. Chen, A dual reciprocity boundary element method for photothermal interactions in laser-induced thermotherapy , International Journal of Heat and Mass Transfer, 51 (2008) Pages 3869-3881.

11. L. Cao, Q.H. Qin, N. Zhao, An RBF-MFS model for analysing thermal behaviour of skin tissues, International Journal of Heat and Mass Transfer, 53 (2010) 1298-1307.

12. M. Jamil, E.Y.K. Ng, Evaluation of meshless radial basis collocation method (RBCM) for heterogeneous conduction and simulation of temperature inside the biological tissues, International Journal of Thermal Sciences 68 (2013) 42-52.

13. W.J. Mansur, C.A.B. Vasconcellos, N.J.M. Zambrozuski, O.C. Rotunno Filho, Numerical solution for linear transient heat conduction using an Explicit Green's Approach. International Journal of Heat and Mass Transfer 52 (2009) 694-701.

14. F.S. Loureiro, W.J. Mansur, C.A.B. Vasconcellos, A Hybrid Time/Laplace Integration Method Based on Numerical Green's Functions in Conduction Heat Transfer, Comput. Meth. Appl. Mech. Eng. 198 (2009) 2662-2672.

15. F.S. Loureiro, W.J. Mansur, A New Family of Time Integration Methods for Heat Conduction Problems Using Numerical Green’s Functions, Computational Mechanics, 44 (2009) 519-531.

16. F.S. Loureiro, W.J. Mansur, A novel higher-order time integration method using the modified explicit Green's approach for linear parabolic problems, Num. Heat Tran., Part B 56 (2009) 211-230.

17. F. S. Loureiro, L. C. Wrobel, W. J. Mansur, Solution of hyperbolic bioheat transfer problems by numerical green's functions: the ExGA-linear $\theta$ method, J. Braz. Soc. Mech. Sci. \& Eng. 34 (2012) 459-468.

18. K.D. Cole, J.V. Beck, A. Haji-Sheikh, B. Litkouhi, Heat conduction using Green’s functions, 2nd Edition. Series in Computational and Physical Processes in Mechanics and Thermal Sciences, Hemisphere Publishing Corporation, 2010.

19. Z.S. Deng, J. Liu, Analytical study on bioheat transfer problems with spatial or transient heating on skin surface or inside biological bodies, ASME J. Biomech Eng. 124 (2002) 638-649.

20. B. Gao, S. Langer, P. M. Corry, Application of the time-dependent Green’s function and Fourier transforms to the solution of the bioheat equation, Int. J. Hyperth. 11 (1995) 267-285. 
21. C.J. Yeung, E. Atalar, A Green's function approach to local rf heating in interventional MRI, Med. Phys. 28 (2001) 826-832.

22. F. Xu, T.J. Lu, Introduction to Skin Biothermomechanics and Thermal Pain. Springer/Science Press, 2010.

23. J. W. Durkee Jr, P. P. Antich, Exact solutions to the multi-region time-dependent bioheat equation with transient heat sources and boundary conditions, Phys. Med. Biol. 36 (1991) 345.

24. M. A. Giordano, G. Gutierrez, C. Rinaldi, Fundamental solutions to the bioheat equation and their application to magnetic fluid hyperthermia, Int J Hyperthermia 26 (2010) 475-484.

25. W. J. Mansur, A Time-stepping Technique to Solve Wave Propagation Problems using the Boundary Element Method, Ph.D. Thesis, University of Southampton, England, 1983.

26. L.C. Wrobel, The boundary element method: applications in thermo-fluids and acoustics, John Wiley \& Sons, England, 2002.

27. T.J.R. Hughes, The finite element method: linear static and dynamic finite element analysis. Prentice-Hall, Englewood Cliffs, New Jersey, 1987.

28. W.L. Wood, Practical time-stepping schemes. Clarendon Press: Oxford, 1990. 
Table 1. Thickness and thermal properties for the layers in the skin model.

\begin{tabular}{|c|c|c|c|c|}
\hline Property & Epidermis & Dermis & Subcutaneous & Muscle \\
\hline $\mathbf{L}_{\mathbf{i}}(\mathbf{m})$ & $8.0 \times 10^{-5}$ & $2.0 \times 10^{-3}$ & $1.0 \times 10^{-2}$ & $3.0 \times 10^{-2}$ \\
\hline $\mathrm{c}\left(\mathrm{J} . \mathrm{kg}^{-1} \cdot{ }^{\mathbf{0}} \mathrm{C}^{-1}\right)$ & $3.6 \times 10^{3}$ & $3.6 \times 10^{3}$ & $2.5 \times 10^{3}$ & $3.8 \times 10^{3}$ \\
\hline$k\left(W \cdot m^{-1} \cdot{ }^{0} C^{-1}\right)$ & 0.22 & 0.4 & 0.2 & 0.45 \\
\hline$\rho\left(\mathrm{kg} \cdot \mathrm{m}^{-3}\right)$ & $1.2 \times 10^{3}$ & $1.2 \times 10^{3}$ & $1.0 \times 10^{3}$ & $1.0 \times 10^{3}$ \\
\hline$C_{b}\left(J^{\prime} \mathrm{kg}^{-1} \cdot{ }^{0} \mathrm{C}^{-1}\right)$ & $4.2 \times 10^{3}$ & $4.2 \times 10^{3}$ & $4.2 \times 10^{3}$ & $4.2 \times 10^{3}$ \\
\hline$\rho_{b}\left(k_{g} \cdot \mathrm{m}^{-3}\right)$ & $1.1 \times 10^{3}$ & $1.1 \times 10^{3}$ & $1.1 \times 10^{3}$ & $1.1 \times 10^{3}$ \\
\hline$w_{b}\left(s^{-1}\right)$ & 0.0 & $5.0 \times 10^{-4}$ & $5.0 \times 10^{-4}$ & $5.0 \times 10^{-4}$ \\
\hline
\end{tabular}




\section{Figure Captions}

Figure 1. Illustration of the two time substeps procedure.

Figure 2. Schematic of a local Green's function discrete domain $\Omega_{G_{j}}^{h}$ for a mesh of four-node quadrilateral elements: a) non-zero values of the Green's function (compact support discrete domain); b) simplified model considering regular elements and taking advantage of the symmetry.

Figure 3. Numerical Green's function $g_{i}^{h}\left(\lambda_{i} \Delta t, \alpha_{1}\right)$ for a SDOF system: a) 3D plot; b) stability region plot.

Figure 4. Comparison of the functions $g_{i}\left(\lambda_{i} \Delta t\right)$ and $g_{i}^{h}\left(\lambda_{i} \Delta t, \alpha_{1}\right)$ for different values of $\alpha_{1}$.

Figure 5. Graph for the accuracy constant $c\left(\alpha_{1}\right)$.

Figure 6. Convergence rate comparison at time $t_{r}=1$ between the explicit Euler method and the ExGA method with $\alpha_{1}=(2-\sqrt{2}) / 4$ and $\alpha_{1}=1 / 2$.

Figure 7. Sketch of the skin burn model: (a) Geometry definitions; b) 2D finite element mesh with 12745 bilinear elements.

Figure 8. Initial temperature field, which is the solution of the steady-state bioheat equation before the heated plate exposure.

Figure 9. Comparative results between the standard Euler scheme and the proposed ExGA method: a) Temperature- time histories at point $(x, y)=(0.00008,0)$; b) Temperature profiles at positions $y=0 \mathrm{~m}, y=0.027 \mathrm{~m}$ and $y=0.03 \mathrm{~m}$ at times $t=294 \mathrm{~s}$ and $t=588 \mathrm{~s}$.

Figure 10. Tissue thermal damage at position $y=0 \mathrm{~m}$ at times $t=294 \mathrm{~s}$ and $t=588 \mathrm{~s}$.

Figure 11. Temperature field at two time instants using the ExGA method: a) $t=294 \mathrm{~s}$; b) $t=588 \mathrm{~s}$. 


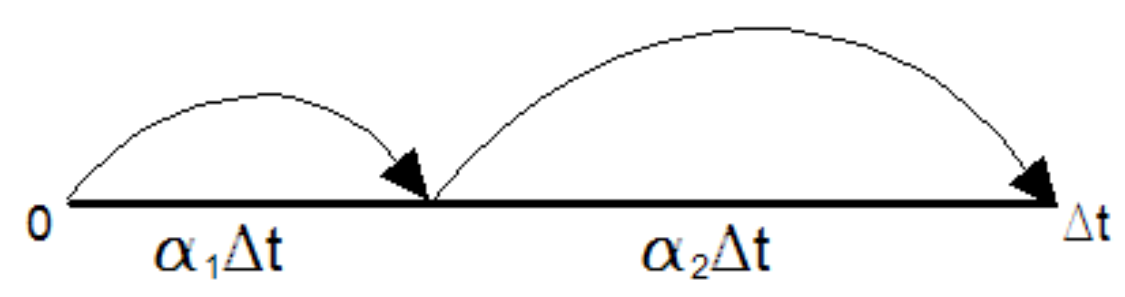

FIGURE 1 


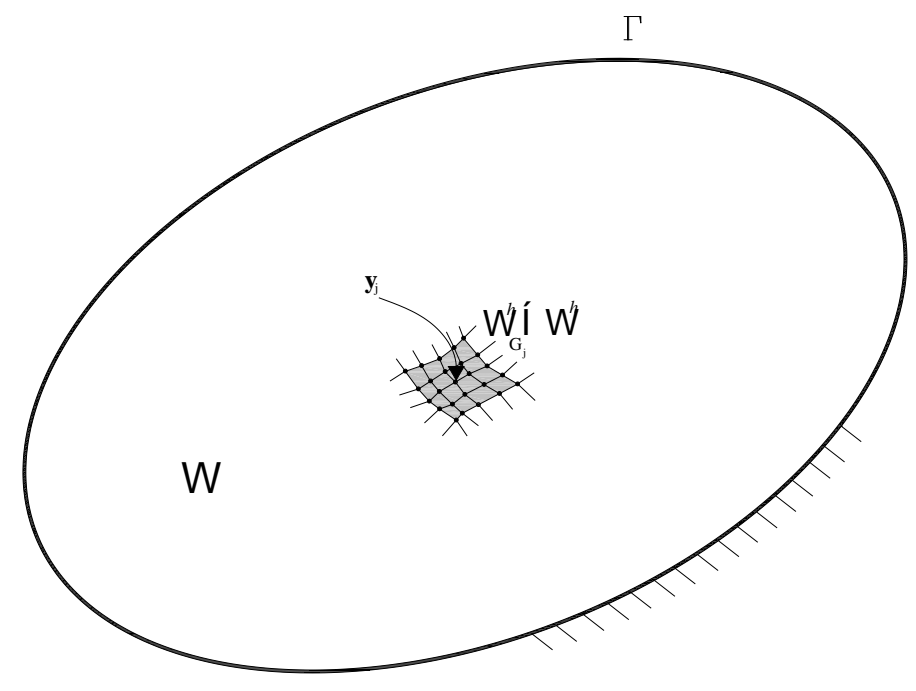

(a)

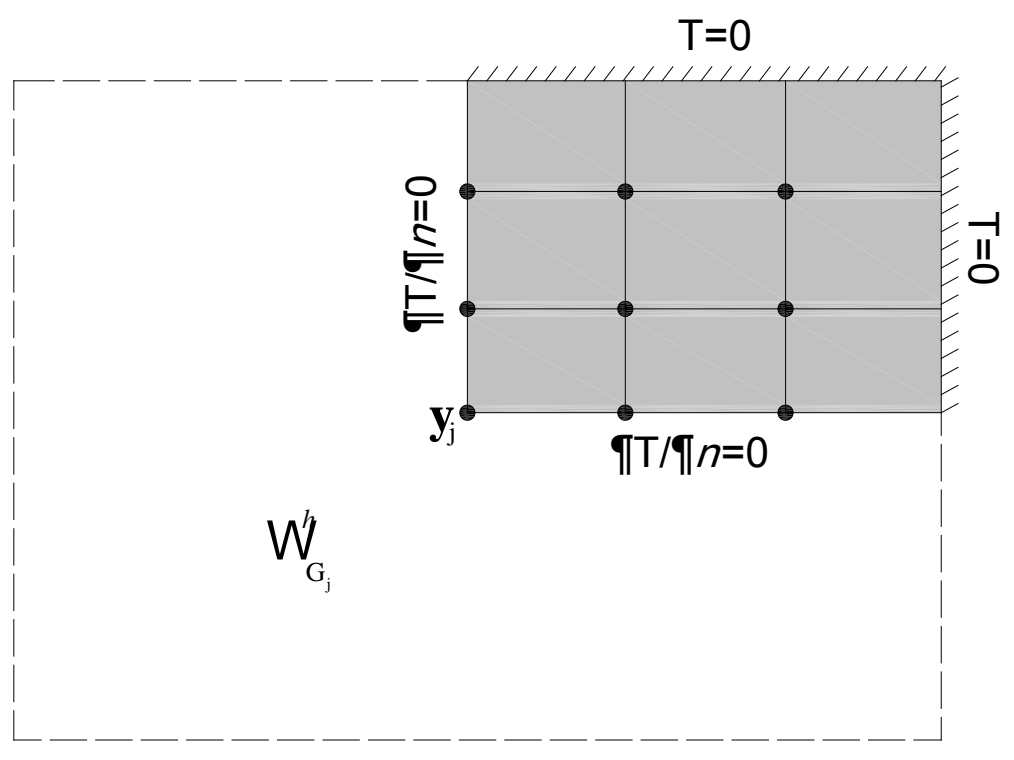

(b)

FIGURE 2 


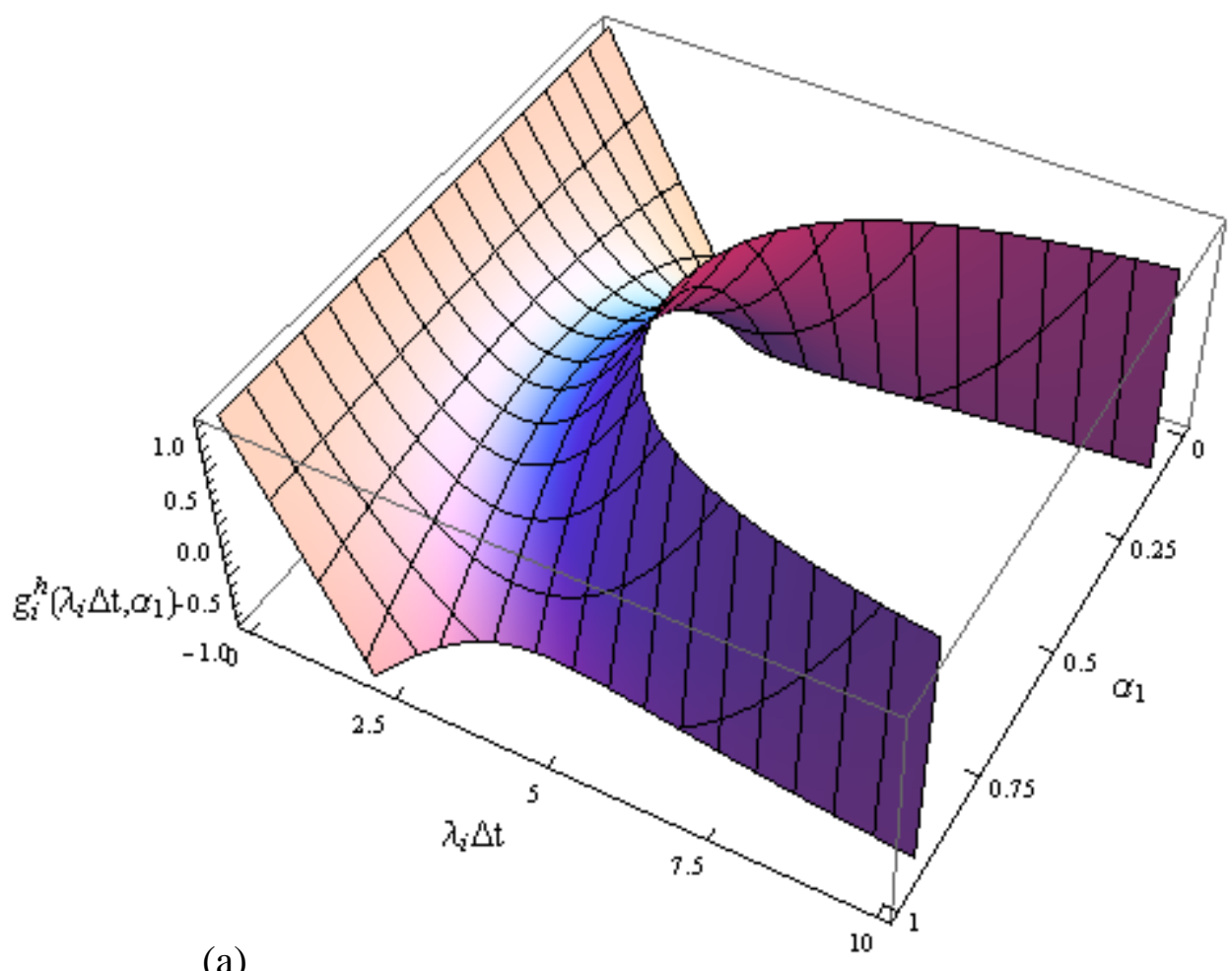

(a)

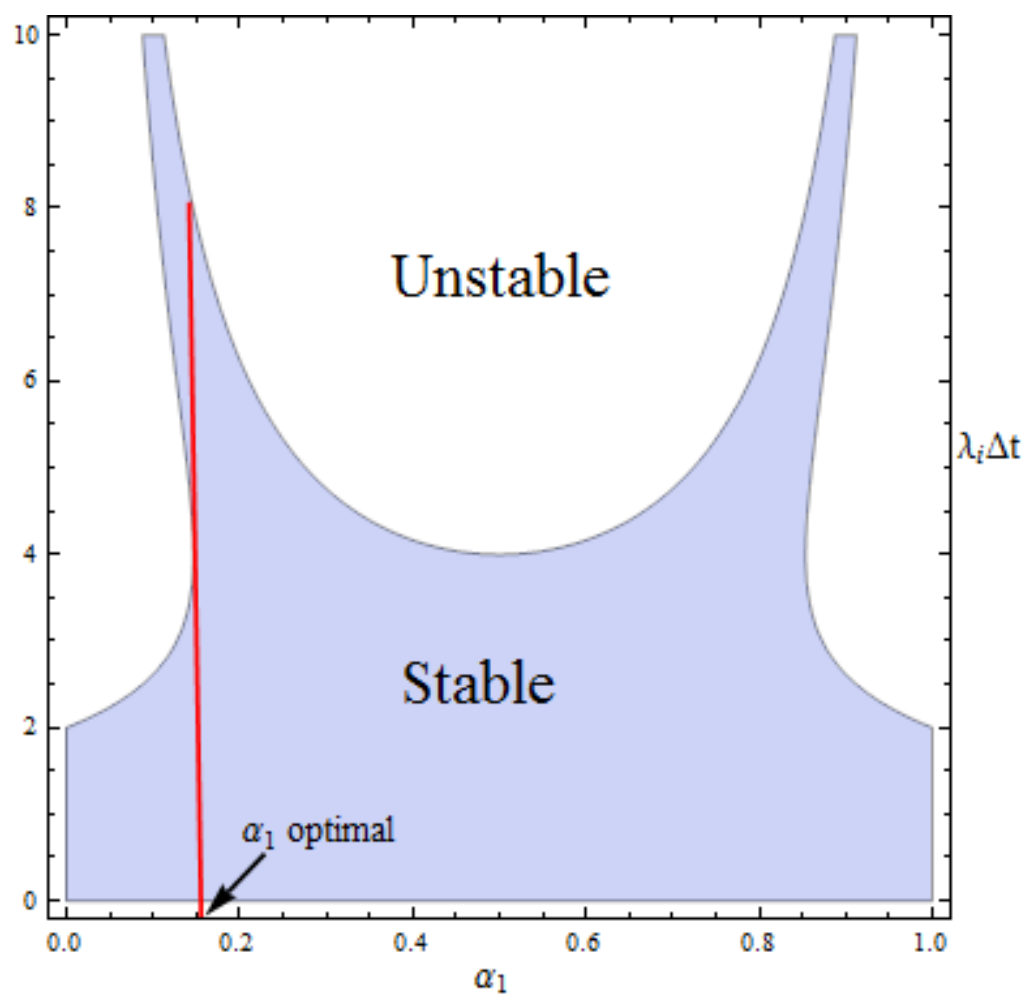

(b)

FIGURE 3 


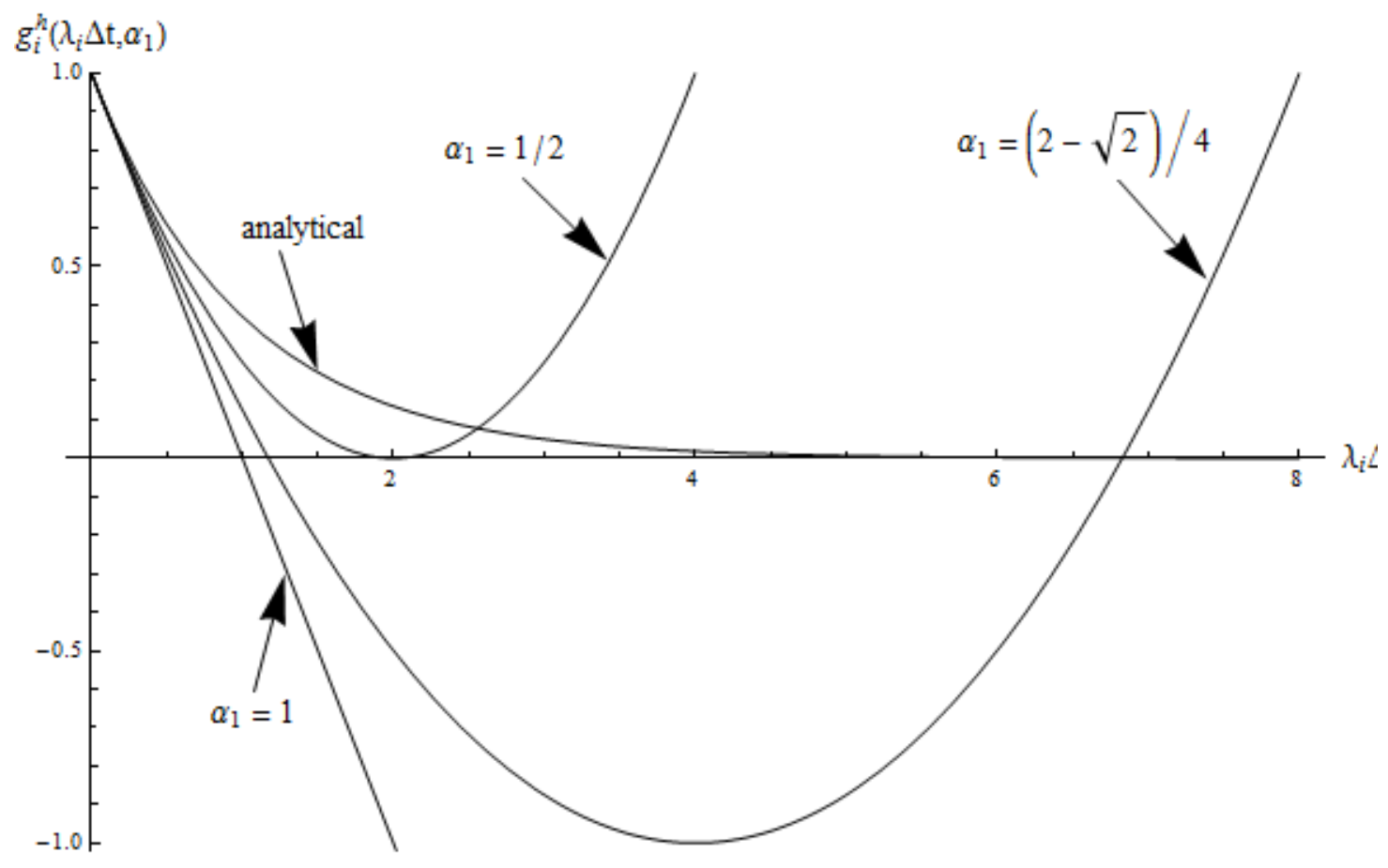

FIGURE 4 


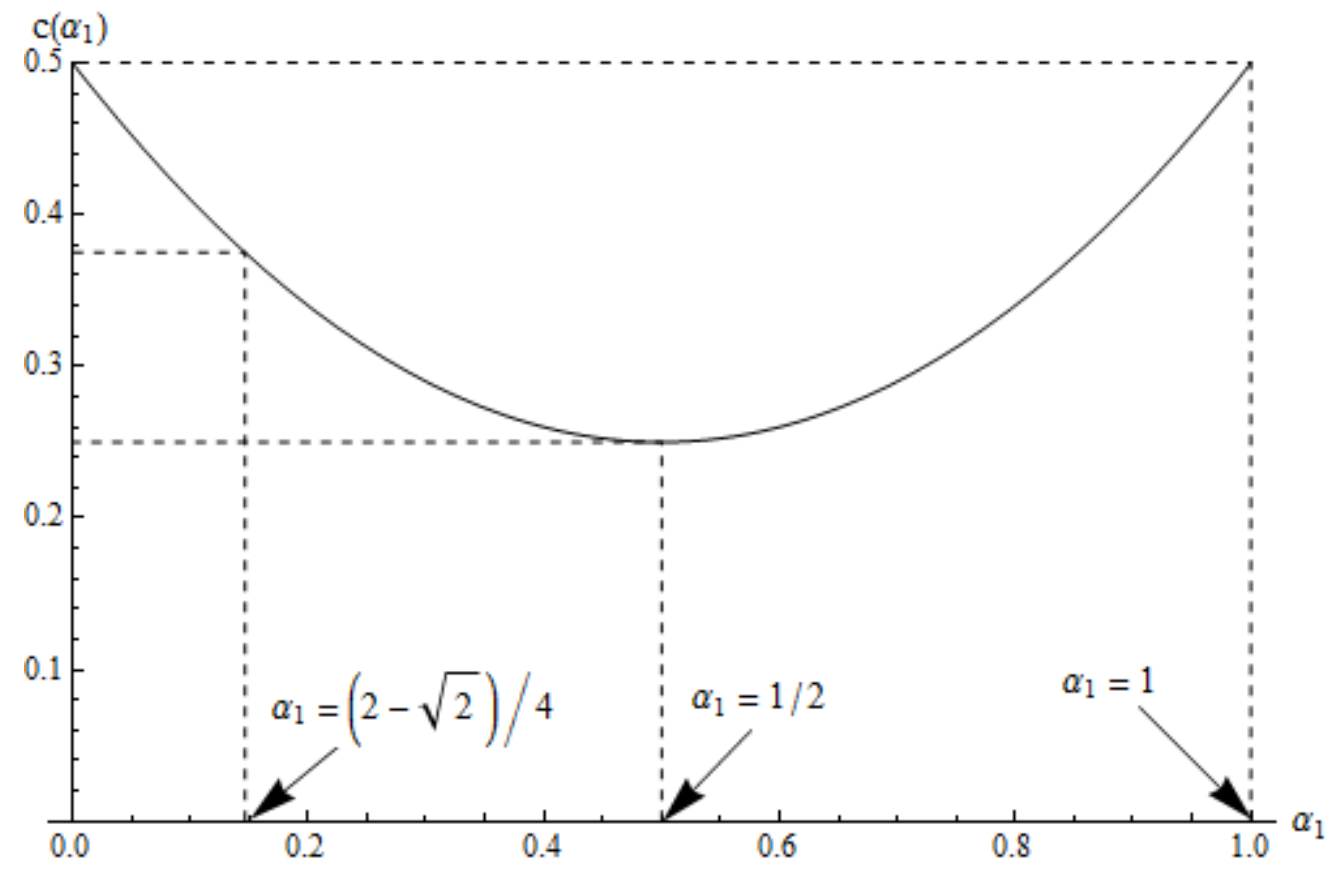

FIGURE 5 


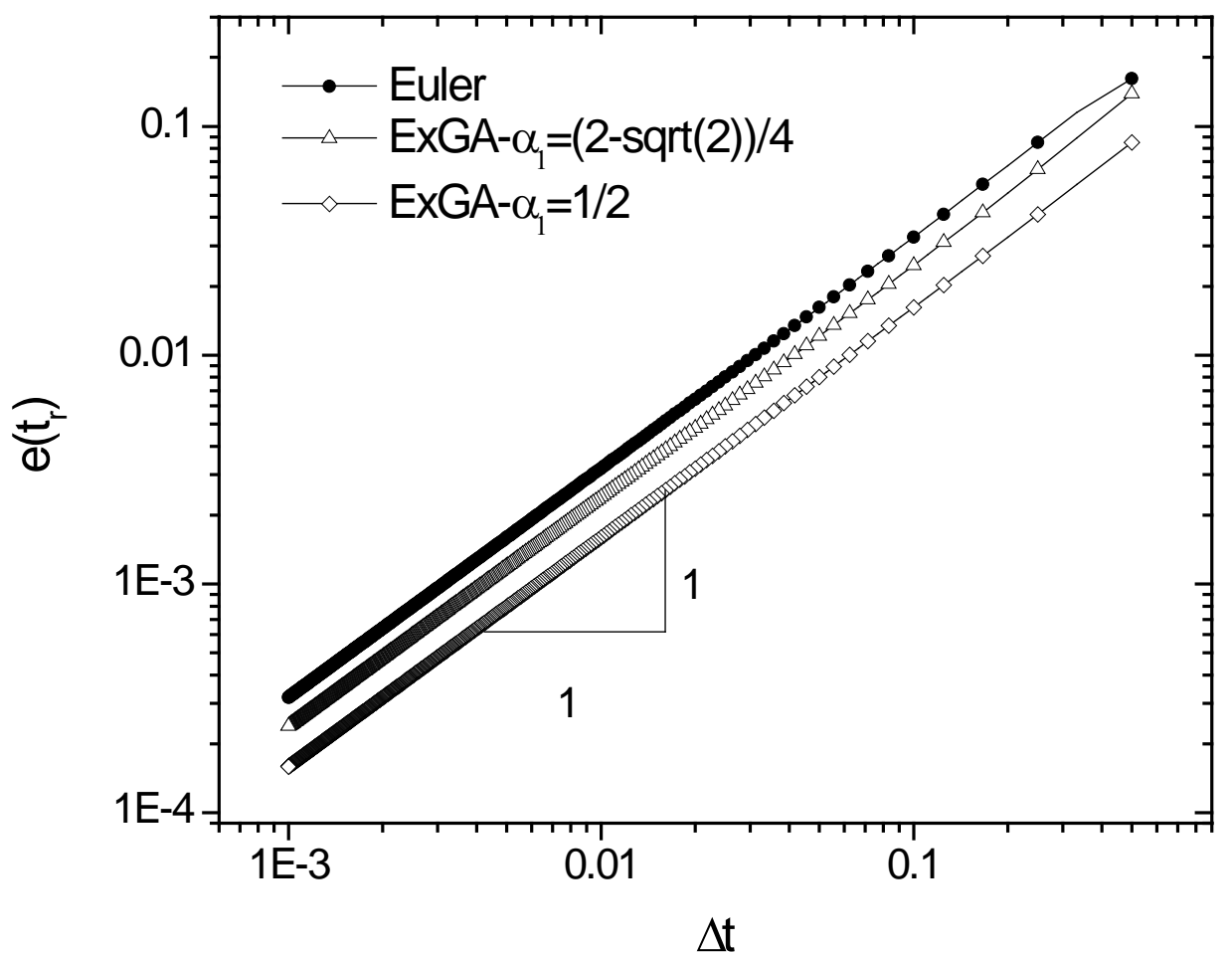

FIGURE 6 


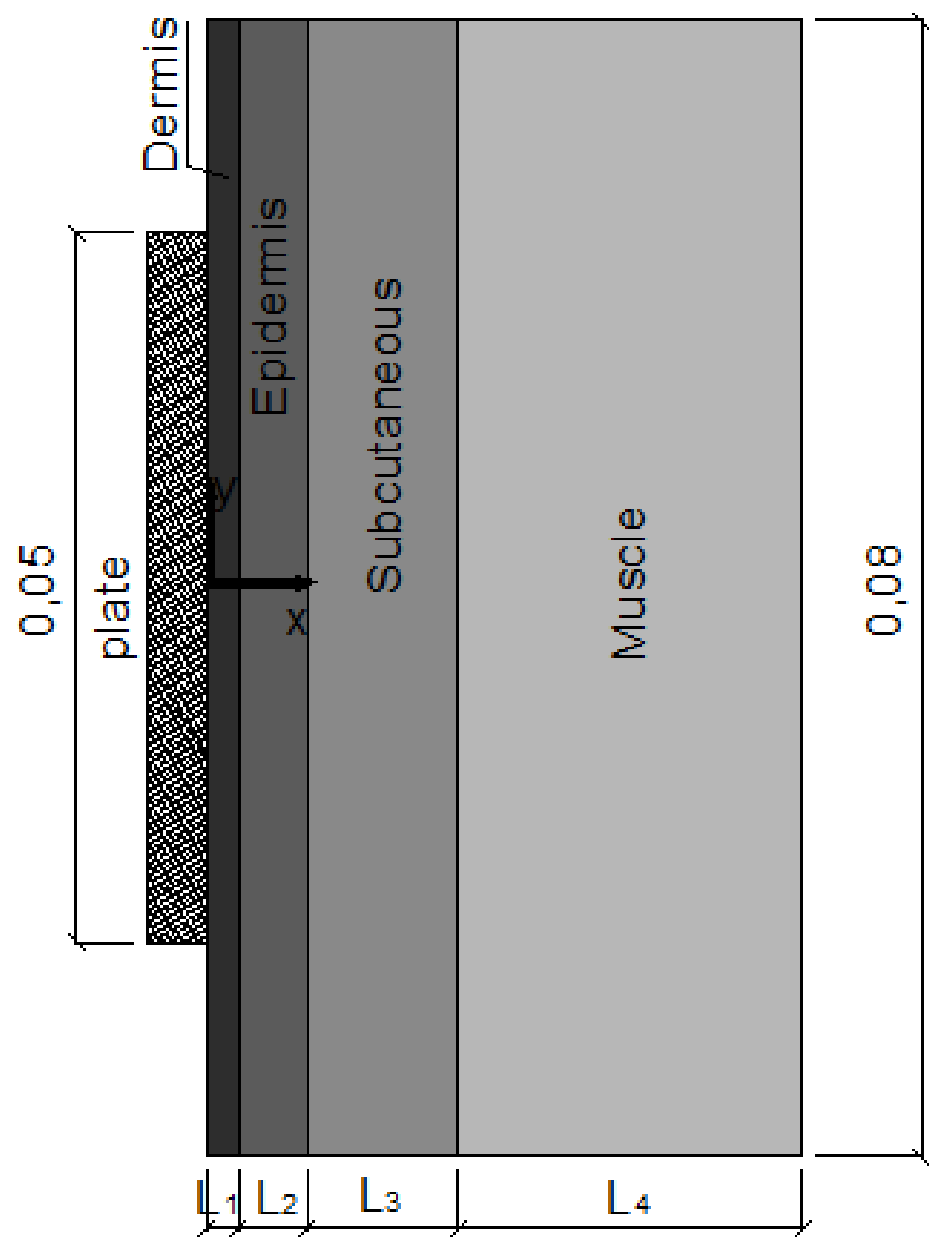

(a)

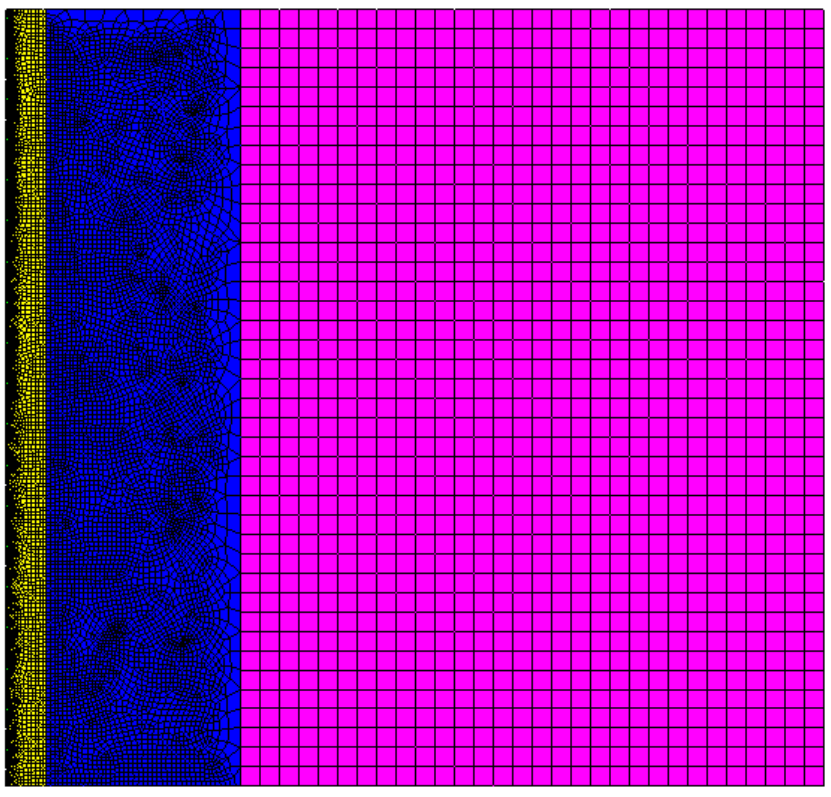

(b) 

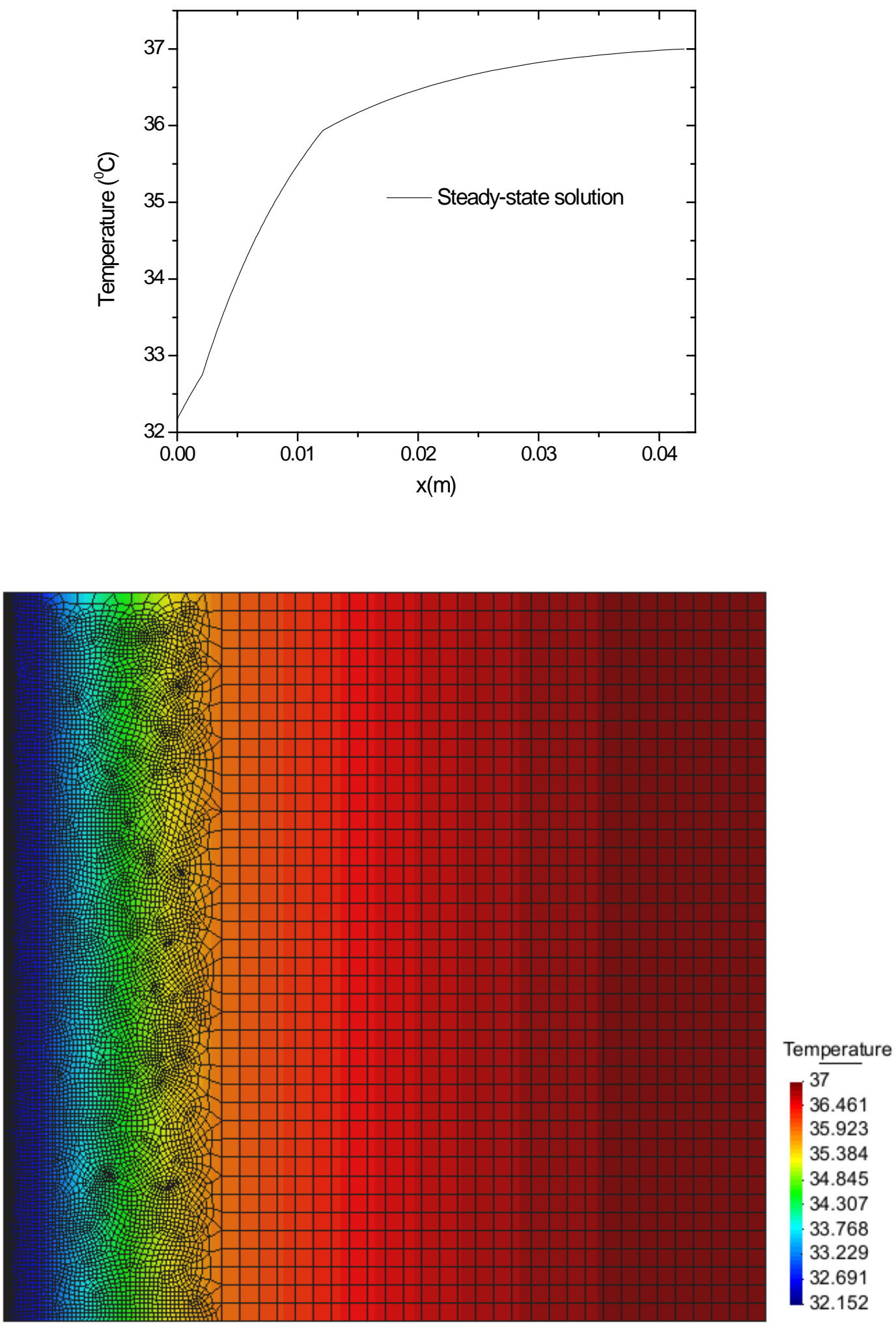

FIGURE 8 


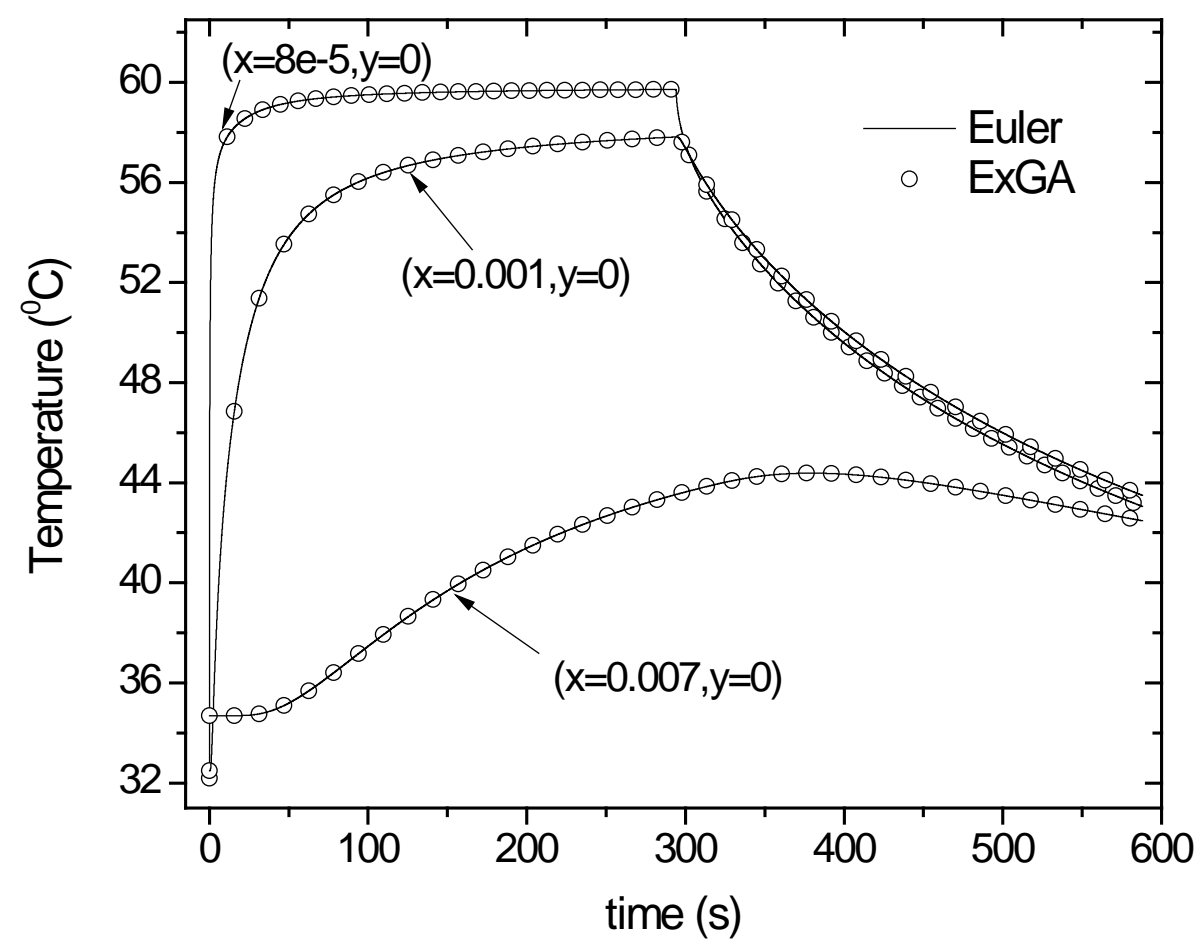

(a)

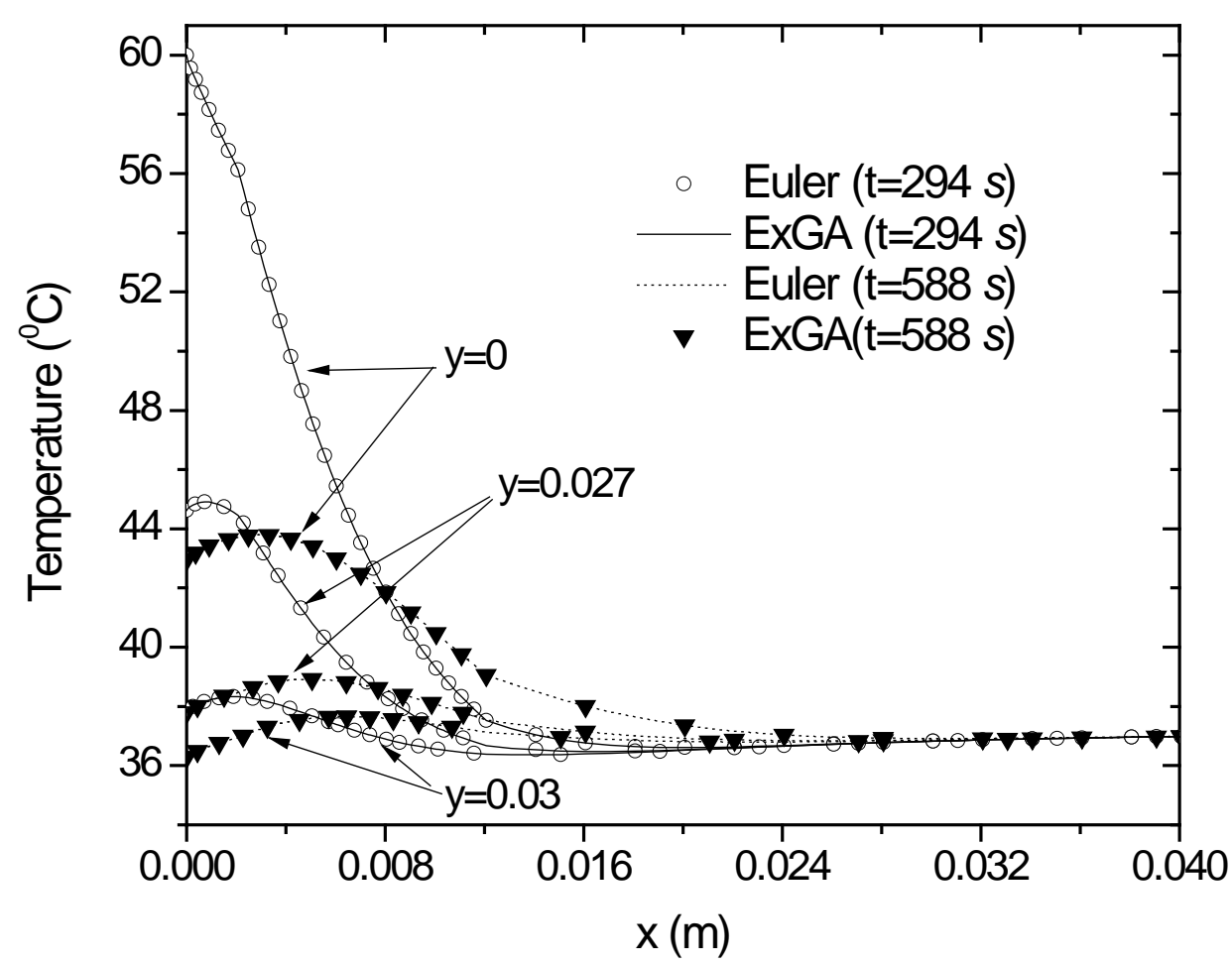

(b)

FIGURE 9 


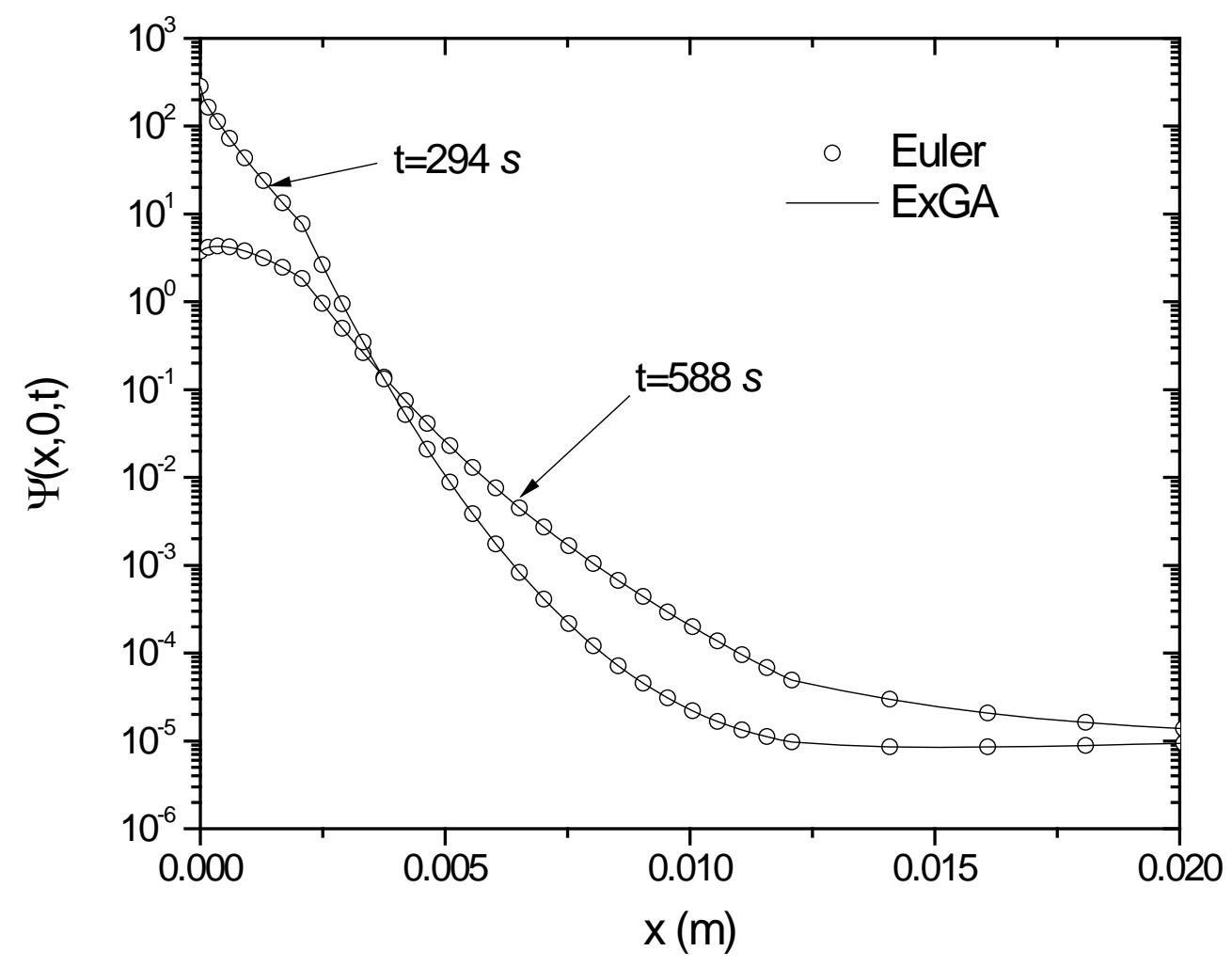

FIGURE 10 


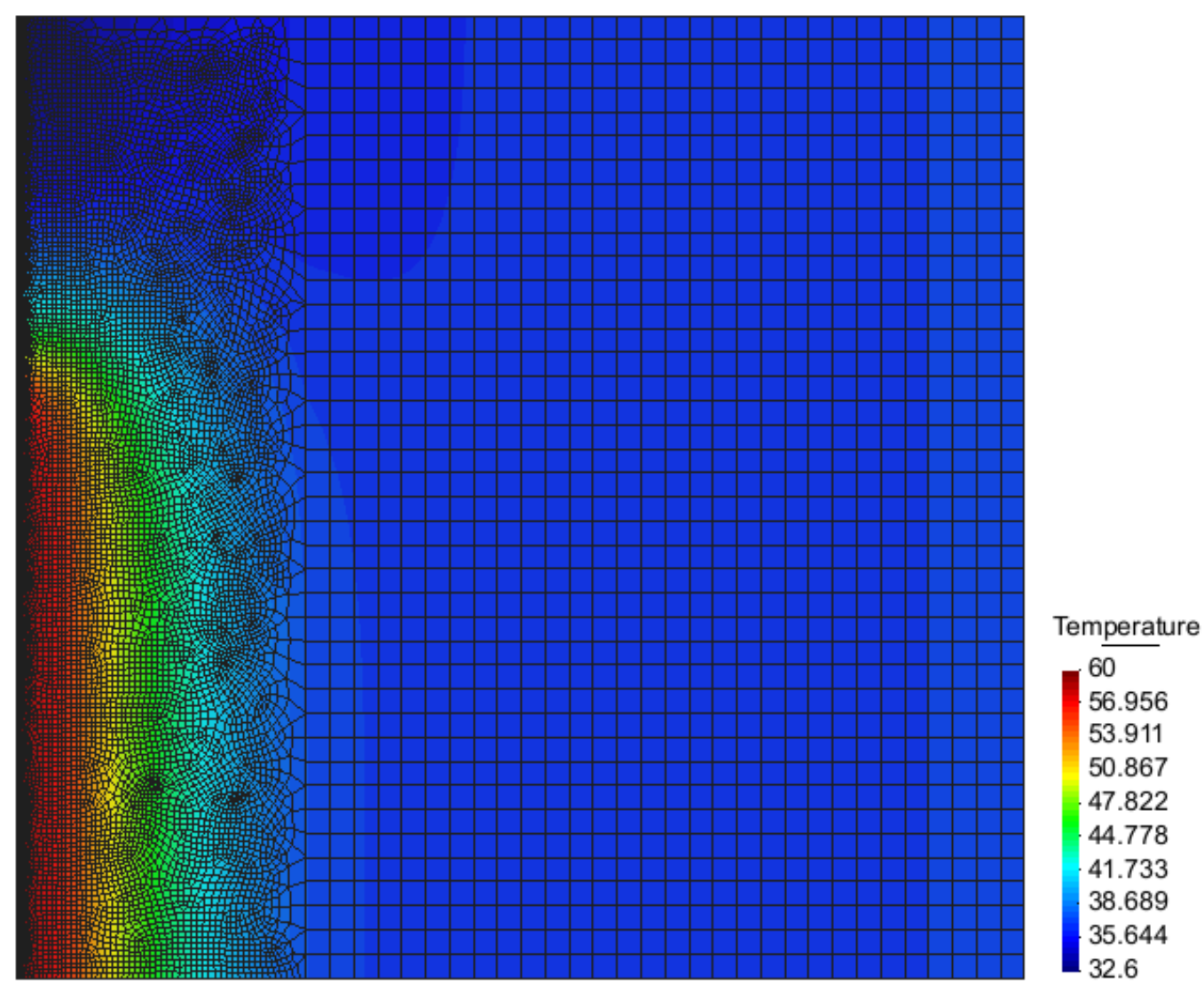

(a)

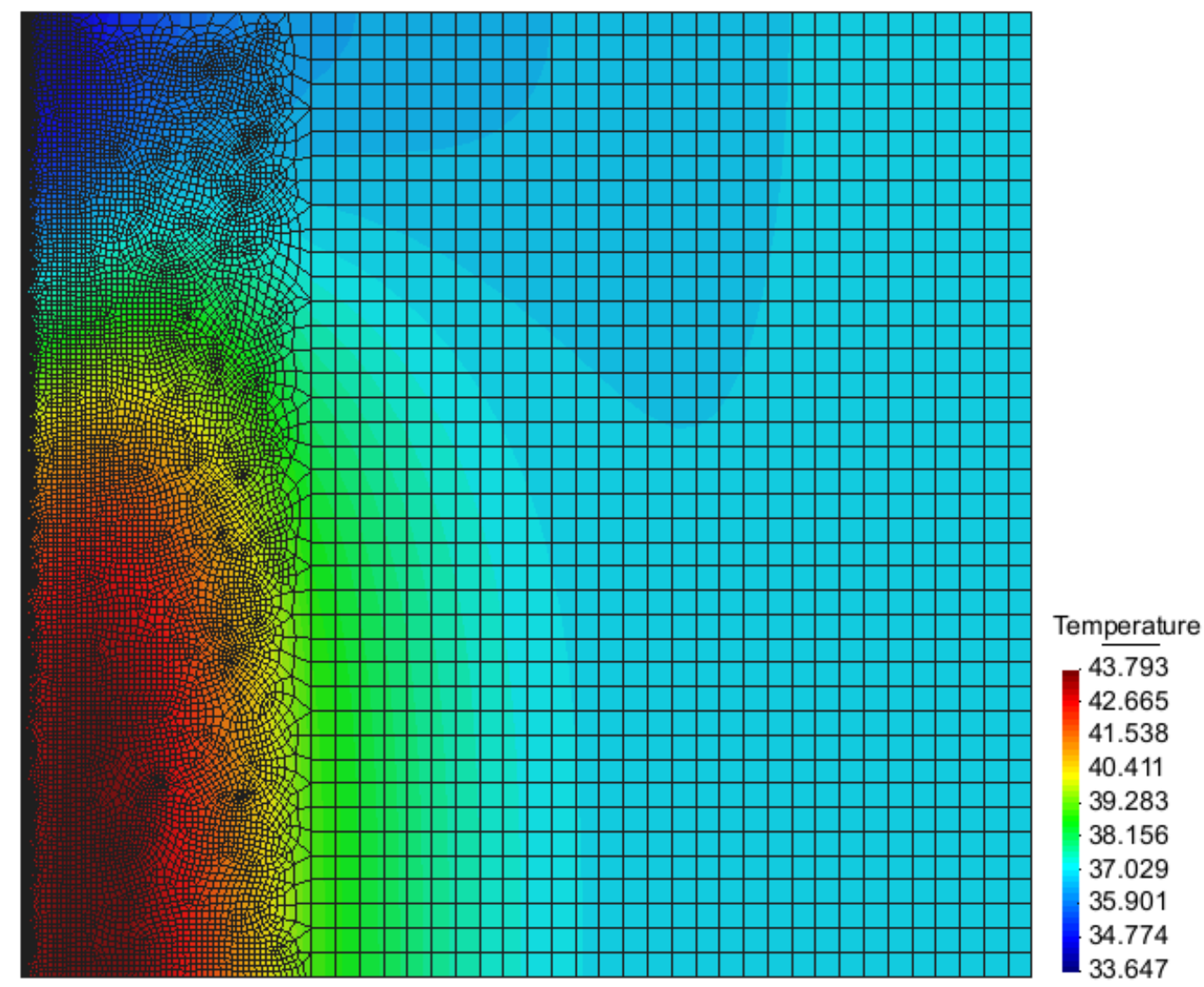

(b) 